\title{
Band alignment of semiconductors from density-functional theory and many-body perturbation theory
}

\section{$\operatorname{AUTHOR}(\mathrm{S}):$}

Hinuma, Yoyo; Grüneis, Andreas; Kresse, Georg; Oba, Fumiyasu

\section{CITATION:}

Hinuma, Yoyo ... [et al]. Band alignment of semiconductors from density-functional theory and many-body perturbation theory. Physical Review B 2014, 90(15): 155405.

ISSUE DATE:

2014-10-03

URL:

http://hdl.handle.net/2433/191210

RIGHT:

(C)2014 American Physical Society 


\title{
Band alignment of semiconductors from density-functional theory and many-body perturbation theory
}

\author{
Yoyo Hinuma, ${ }^{1, *}$ Andreas Grüneis, ${ }^{2}$ Georg Kresse,${ }^{2}$ and Fumiyasu Oba ${ }^{1,3, \dagger}$ \\ ${ }^{1}$ Department of Materials Science and Engineering, Kyoto University, Kyoto 606-8501, Japan \\ ${ }^{2}$ Faculty of Physics and Center for Computational Materials Science, University of Vienna, Sensengasse 8/12, A-1090 Vienna, Austria \\ ${ }^{3}$ Materials Research Center for Element Strategy, Tokyo Institute of Technology, Yokohama 226-8503, Japan
}

(Received 12 June 2014; revised manuscript received 11 September 2014; published 3 October 2014)

\begin{abstract}
The band lineup, or alignment, of semiconductors is investigated via first-principles calculations based on density functional theory (DFT) and many-body perturbation theory (MBPT). Twenty-one semiconductors including $\mathrm{C}, \mathrm{Si}$, and $\mathrm{Ge}$ in the diamond structure, BN, AlP, AlAs, AlSb, GaP, GaAs, GaSb, InP, InAs, InSb, $\mathrm{ZnS}, \mathrm{ZnSe}, \mathrm{ZnTe}, \mathrm{CdS}, \mathrm{CdSe}$, and CdTe in the zinc-blende structure, and $\mathrm{GaN}$ and $\mathrm{ZnO}$ in the wurtzite structure are considered in view of their fundamental and technological importance. Band alignments are determined using the valence and conduction band offsets from heterointerface calculations, the ionization potential (IP) and electron affinity (EA) from surface calculations, and the valence band maximum and conduction band minimum relative to the branch point energy, or charge neutrality level, from bulk calculations. The performance of various approximations to DFT and MBPT, namely the Perdew-Burke-Ernzerhof (PBE) semilocal functional, the Heyd-Scuseria-Ernzerhof (HSE) hybrid functional, and the $G W$ approximation with and without vertex corrections in the screened Coulomb interaction, is assessed using the $G W \Gamma^{1}$ approximation as a reference, where first-order vertex corrections are included in the self-energy. The experimental IPs, EAs, and band offsets are well reproduced by $G W \Gamma^{1}$ for most of the semiconductor surfaces and heterointerfaces considered in this study. The PBE and HSE functionals show sizable errors in the IPs and EAs, in particular for group II-VI semiconductors with wide band gaps, but are much better in the prediction of relative band positions or band offsets due to error cancellation. The performance of the $G W$ approximation is almost on par with $G W \Gamma^{1}$ as far as relative band positions are concerned. The band alignments based on average interfacial band offsets for all pairs of 17 semiconductors and branch point energies agree with explicitly calculated interfacial band offsets with small mean absolute errors of both $\sim 0.1 \mathrm{eV}$, indicating a good overall transitivity of the band offsets. The alignment based on IPs from selected nonpolar surfaces performs comparably well in the prediction of band offsets at most of the considered interfaces. The maximum errors are, however, as large as $0.3,0.4$, and $0.7 \mathrm{eV}$ for the alignments based on the average band offsets, branch point energies, and IPs, respectively. This margin of error should be taken into account when performing materials screening using these alignments.
\end{abstract}

DOI: 10.1103/PhysRevB.90.155405

PACS number(s): 73.20.At, 73.40.Kp, 73.40.Lq

\section{INTRODUCTION}

The band lineup, or alignment, carries fundamental information relevant to the design of a wide variety of semiconductors and their surfaces and heterojunctions, including the prediction of the Schottky barrier heights of metalsemiconductor interfaces, the band offsets of semiconductor heterointerfaces, the band positions with respect to vacuum and adsorbate levels at surfaces, and even doping limits [1-5]. The alignment should in principle be optimized for target quantities and properties, which can be surface or interface structure dependent. However, a single and sufficiently universal alignment diagram may be used to predict many kinds of functionalities, at least for initial materials screening. The construction of a reliable band alignment from both theory and experiment has thus been a long-standing issue because of its fundamental and technological importance.

Historically, various theories have been proposed in connection with the band alignment of semiconductors, particularly, for the prediction of band offsets at heterojunctions [1-4].

\footnotetext{
*yoyo.hinuma@gmail.com

†oba@cms.mtl.kyoto-u.ac.jp
}

These include the LCAO (linear combination of atomic orbitals) theory [6], the model solid theory [7], the branch point energy or the charge neutrality level [8-10], the dielectric midgap energy [11], and the self-consistent dipole theory [12], most of which have been used in combination with first-principles calculations. More recent first-principles approaches to band alignments can be broadly separated into four categories. The first involves explicit modeling of heterointerfaces to obtain unstrained, or natural, valence and conduction band offsets between two materials. A band alignment diagram assuming transitivity can be constructed by averaging the offset values for a large number of heterointerfaces $[7,13]$. The second uses branch point energies or charge neutrality levels, which are determined solely from bulk band structures, to predict interfacial band offsets $[4,8,14,15]$. The third is based on the transition levels of impurities such as hydrogen [16] and transition-metal elements [17,18]. The fourth aligns the valence and conduction bands of surfaces against the vacuum level [19-22]. The ionization potential (IP) and electron affinity (EA) representing the valence band maximum (VBM) and conduction band minimum (CBM) with respect to the vacuum level, respectively, are key quantities that largely determine a variety of surface properties. The IP or EA differences can be used to estimate interfacial band offsets, 
assuming that the difference in the dipole contributions at two surfaces well approximates the contribution of interfacial dipoles.

An accurate determination of band offsets at heterojunctions is relevant to virtually all electronic devices and photovoltaic cells using semiconductor and/or insulator heterostructures. The valence and conduction band offsets denote the discontinuity in the VBM and CBM, respectively, between two semiconductors or insulators that constitute an interface [1,2]. A number of experimental and theoretical studies have been conducted to evaluate the band offsets $[3,4,7,8,13,23-26]$. The band offsets are affected by the interface dipole that depends on the interfacial orientation and atomic configuration. Such a dipole contribution, however, could be small when the phases that constitute the interface have similar atomic arrangements and chemical bonding states. As a result, band alignments assuming transitivity of band offsets $[7,13]$ are often useful.

Direct modeling of interfaces is a straightforward approach to the evaluation of interfacial band offsets. An inevitable problem is that most of heterointerfaces in semicoherent structures with misfit dislocations and incoherent structures cannot be readily modeled in view of the diversity and complication in the atomic structure. In addition, large simulation models and hence large computational costs are incurred. In many cases, lattice-matched, or coherent, interfaces, where in-plane lattice parameters (ILPs) are the same in the two constituent phases, are treated under three-dimensional periodic boundary conditions. This approach is appropriate to an actually coherent interface, such as the interface between a substrate and a strained epitaxial film of a second phase below the critical thickness [27]. However, corrections need to be made regarding the ILPs to obtain the band offsets between unstrained constituent phases, namely the "unstrained" or "natural" band offsets.

The branch point energy is the energy where the character of electronic states changes from valence bandlike to conduction bandlike and can act as the charge neutrality level [4,8-10]. Tersoff predicted interfacial band offsets via alignment at the branch point energies, assuming that the bands of two semiconductors tend to align so that interfacial dipoles vanish as a consequence of dipolar response [8,9]. This approach has been successful for various elementary and compound semiconductors $[4,8,9,14,15,22]$. It is also noteworthy that the branch point energy-based or charge neutrality level-based alignment is computationally efficient because only bulk calculations are required.

The band alignment can be determined using the IP and/or $\mathrm{EA}$, for which the vacuum level serves as a common reference $[4,5,20,21,28-30]$. The IP and EA depend on the surface orientation and atomic structures and, in addition, are affected by adsorption or contamination of the surfaces. These changes are relevant to the design of surface functionalities such as photocatalysis, where the surface structure and adsorbate coverage can be tuned to modify the IP and EA and thereby change the reactivity [19]. There can be at least two definitions of the IP and EA: Some definitions explicitly include the effects of surface localized states [31,32] while the "bulk-based" IP is defined as the difference of the vacuum level and the VBM in the bulklike region far from the surface [32]. The latter is more suitable for the determination of band alignments.
The theoretical evaluation of interfacial band offsets, branch point energies, IPs, EAs, and the construction of their resultant band alignments have been made via first-principles calculations, mostly using the local density approximation (LDA) [33,34] and the generalized gradient approximation (GGA) [35-37] to density functional theory (DFT) [38]. The accuracy of the predictions depends strongly on the applied approximation, as reported for the IPs of prototypical semiconductors for which experimental values are relatively well established [21]. Hybrid functionals, which mix the exact Hartree-Fock exchange with LDA or GGA functionals, have been shown to significantly improve the electronic structure of semiconductors and insulators over the local and semilocal functionals [39-41]. These functionals have also been successfully applied to their lattice defects including point defects [42-50], surfaces [21,31,32,48,51], and heterointerfaces [52-54]. Still, noticeable errors prevail in the band gaps and IPs, in particular, of wide gap materials [21,40]. The $G W$ approximation to many-body perturbation theory (MBPT) [55,56] in general predicts the band structure of solids more accurately and has also been applied to the evaluation of IPs and EAs [19-22], interfacial band offsets [26,57-60], and branch point energies [15,22]. We have recently reported that the $G W$ approximation suffers from sizable self-interaction errors not only for localized states, as pointed out previously $[61,62]$, but also for extended states in the valence and conduction bands of most semiconductors [21]. The $G W \Gamma^{1}$ approximation that includes the first-order vertex correction in the self-energy improves the band positions, leading to better agreement with experiments for IPs [21]. It is therefore important to revisit the band alignment of semiconductors using $G W \Gamma^{1}$ as a reference.

The objective of this work is to investigate the band alignment of semiconductors based on three approaches using valence and conduction band offsets from heterointerface calculations, IPs and EAs from surface calculations, and VBMs and CBMs relative to branch point energies from bulk calculations. Twenty-one semiconductors in diamond $(\mathrm{C}, \mathrm{Si}$, and $\mathrm{Ge}$ ), zinc-blende (BN, AlP, AlAs, AlSb, GaP, GaAs, $\mathrm{GaSb}, \mathrm{InP}, \mathrm{InAs}, \mathrm{InSb}, \mathrm{ZnS}, \mathrm{ZnSe}, \mathrm{ZnTe}, \mathrm{CdS}, \mathrm{CdSe}$, and $\mathrm{CdTe})$, and wurtzite ( $\mathrm{GaN}$ and $\mathrm{ZnO})$ structures are selected in view of the availability of relatively accurate and large numbers of experimental offset, IP, and EA values, as well as their technological importance in electronic and optoelectronic devices and photovoltaic cells. The performance of a variety of approximations including semilocal and hybrid functionals and $G W$ approximations is assessed using $G W \Gamma^{1}$ as a reference. The band alignments based on average interfacial band offsets, IPs and EAs, and branch point energies are discussed in connection with the prediction of interfacial band offsets.

\section{METHODOLOGY}

\section{A. Computational procedures}

The IPs, EAs, valence and conduction band offsets, and branch point energies are obtained via surface, interface, and bulk calculations and their combinations. The calculations are performed in the framework of the generalized Kohn-Sham 
TABLE I. Experimental lattice constants $(a$ and $c)$, band gaps $\left(E_{\mathrm{g}}^{\text {expt }}\right)$, and theoretical band gaps obtained using various approximations $\left(E_{\mathrm{g}}^{\text {theory }}\right)$ for semiconductors in the diamond, zinc-blende, and wurtzite structures considered in this study. The effects of spin-orbit coupling are included as a correction in the theoretical band gaps. The experimental values and the theoretical values other than the PBE band gaps are collected from Ref. [21] and references therein. The lattice constant ratio versus $\mathrm{ZnS}$ is also shown for diamond and zinc-blende phases.

\begin{tabular}{|c|c|c|c|c|c|c|c|c|c|}
\hline & \multirow[b]{2}{*}{$a(\AA)$} & \multirow[b]{2}{*}{$c(\AA)$} & \multirow[b]{2}{*}{ Ratio } & \multirow[b]{2}{*}{$E_{\mathrm{g}}^{\text {expt }}(\mathrm{eV})$} & \multicolumn{5}{|c|}{$E_{\mathrm{g}}^{\text {theory }}(\mathrm{eV})$} \\
\hline & & & & & $\mathrm{PBE}$ & HSE & $G W_{0} @ \mathrm{PBE}$ & $G W^{\mathrm{TC}-\mathrm{TC}} @ \mathrm{HSE}$ & $\overline{G W \Gamma^{1} @ \mathrm{HSE}}$ \\
\hline $\mathrm{C}$ & 3.567 & - & $65.9 \%$ & 5.48 & 4.15 & 5.34 & 5.81 & 5.83 & 5.92 \\
\hline $\mathrm{BN}$ & 3.616 & - & $66.9 \%$ & 6.25 & 4.46 & 5.80 & 6.66 & 6.63 & 6.81 \\
\hline $\mathrm{ZnS}$ & 5.409 & - & $100.0 \%$ & 3.6 & 2.09 & 3.29 & 3.72 & 3.81 & 3.90 \\
\hline $\mathrm{Si}$ & 5.431 & - & $100.4 \%$ & 1.17 & 0.69 & 1.15 & 1.21 & 1.26 & 1.35 \\
\hline $\mathrm{GaP}$ & 5.451 & - & $100.8 \%$ & 2.26 & 1.63 & 2.28 & 2.40 & 2.45 & 2.53 \\
\hline AlP & 5.463 & - & $101.0 \%$ & 2.45 & 1.56 & 2.27 & 2.62 & 2.64 & 2.72 \\
\hline GaAs & 5.654 & - & $104.5 \%$ & 1.52 & 0.48 & 1.31 & 1.21 & 1.48 & 1.54 \\
\hline $\mathrm{Ge}$ & 5.658 & - & $104.6 \%$ & 0.74 & 0 & 0.72 & 0.55 & 0.78 & 0.84 \\
\hline AlAs & 5.661 & - & $104.7 \%$ & 2.16 & 1.32 & 1.99 & 2.35 & 2.38 & 2.45 \\
\hline $\mathrm{ZnSe}$ & 5.669 & - & $104.8 \%$ & 2.7 & 1.18 & 2.30 & 2.66 & 2.86 & 2.93 \\
\hline $\mathrm{CdS}$ & 5.818 & - & $107.6 \%$ & 2.42 & 1.15 & 2.16 & 2.35 & 2.52 & 2.59 \\
\hline InP & 5.869 & - & $108.5 \%$ & 1.42 & 0.75 & 1.50 & 1.33 & 1.53 & 1.58 \\
\hline InAs & 6.058 & - & $112.0 \%$ & 0.42 & 0 & 0.40 & 0.25 & 0.47 & 0.50 \\
\hline $\mathrm{CdSe}$ & 6.077 & - & $112.3 \%$ & 1.73 & 0.51 & 1.44 & 1.60 & 1.86 & 1.91 \\
\hline $\mathrm{GaSb}$ & 6.096 & - & $112.7 \%$ & 0.81 & 0 & 0.68 & 0.51 & 0.77 & 0.80 \\
\hline $\mathrm{ZnTe}$ & 6.103 & - & $112.8 \%$ & 2.25 & 0.96 & 1.99 & 2.15 & 2.36 & 2.42 \\
\hline $\mathrm{AlSb}$ & 6.136 & - & $113.4 \%$ & 1.65 & 0.99 & 1.56 & 1.76 & 1.78 & 1.84 \\
\hline $\mathrm{InSb}$ & 6.479 & - & $119.8 \%$ & 0.24 & 0 & 0.25 & 0.11 & 0.31 & 0.33 \\
\hline $\mathrm{CdTe}$ & 6.481 & - & $119.8 \%$ & 1.45 & 0.49 & 1.35 & 1.44 & 1.66 & 1.70 \\
\hline$w-\mathrm{GaN}$ & 3.190 & 5.186 & - & 3.4 & 2.00 & 3.19 & 3.48 & 3.62 & 3.75 \\
\hline$w-\mathrm{ZnO}$ & 3.250 & 5.207 & - & 3.4 & 0.80 & 2.55 & 3.40 & 3.68 & 3.88 \\
\hline
\end{tabular}

scheme $[63,64]$ using the projector augmented-wave (PAW) method [65] and either the Perdew-Burke-Ernzerhof (PBE) semilocal GGA functional [35,36] or the Heyd-ScuseriaErnzerhof (HSE) hybrid functional [39,66,67] as implemented in the VASP code [40,68-70]. The $G W$ and $G W \Gamma^{1}$ approximations are also applied in the bulk to determine many-body corrections to the VBM and CBM; surface and interface calculations are conducted only at the PBE and HSE levels, as detailed later. The effect of spin-orbit coupling is evaluated for the bulk using HSE and included as a correction to the VBM in all of the PBE, HSE, $G W$, and $G W \Gamma^{1}$ results [21].

The bulk calculations are carried out using primitive cells with experimental lattice parameters listed in Table I for a direct comparison with experimental IPs, EAs, and band offsets. A plane wave cutoff energy of $550 \mathrm{eV}$ and $6 \times 6 \times 6 k$ points are used in the PBE and HSE calculations. Subsequent $G W$ calculations are performed on top of PBE or HSE using PAW potentials with approximately norm-conserving partial waves [21,71]. The plane wave cutoff energies are increased up to $\sim 1200 \mathrm{eV}$, including all unoccupied orbitals, and the results are extrapolated to the infinite basis set limit [71,72]. In the $G W$ calculations, orbitals are kept fixed so that the electrostatic alignment between the bulk and surfaces or interfaces can be readily made via the local Kohn-Sham potential determined at either the PBE or HSE level.

Three types of approximations, climbing up the hierarchy in the following order, are used. The first one is $G W_{0} @ \mathrm{PBE}$, which is a commonly taken $G W$ approach. Using PBE orbitals, the quasiparticle (QP) energies in the Green's function $G$ are iteratively updated to self-consistency. The screened
Coulomb interaction $W$ is determined using the random phase approximation (RPA) and PBE one-electron energy differences in the independent particle polarizability; $W$ is not updated $\left(W_{0}\right)$. The second one is $G W^{\mathrm{TC}-\mathrm{TC}} @ \mathrm{HSE}$, or simply $G W^{\mathrm{TC}-\mathrm{TC}}$, where HSE orbitals are used, vertex corrections are self-consistently included in $W$ using the static approximation $\left[W^{\mathrm{TC}-\mathrm{TC}}\right.$, denoting the test charge-test charge (TC-TC) screened Coulomb interaction], and the QP energies are updated both in $G$ and in the independent particle polarizability. This approach has been shown to yield very similar screening properties to the RPA with PBE one-electron energies for a number of semiconductors, i.e., $W_{0} @ \mathrm{PBE} \approx W^{\mathrm{TC}-\mathrm{TC}} @ \mathrm{HSE}$ [73], but $W^{\mathrm{TC}-\mathrm{TC}} @$ HSE is physically better justified: In this approach the QP energies are roughly at the experimental energies, and electron-hole and excitonic interactions are accounted for. The third approximation is $G W \Gamma^{1} @ \mathrm{HSE}$, or simply $G W \Gamma^{1}$, where the first-order vertex correction in the self-energy is included as described in Ref. [21]. The $G W^{\mathrm{TC}-\mathrm{TC}} @$ HSE results are used as a starting point and the self-energy vertex corrections are added $a$ posteriori. This contribution is evaluated using $4 \times 4 \times 4 k$ points, while the $G W_{0} @ \mathrm{PBE}$ and $G W^{\mathrm{TC}-\mathrm{TC}}$ calculations are conducted using $6 \times 6 \times 6 k$ points.

Nonpolar surfaces and interfaces are exclusively considered in this study, because their structures are relatively well established. For polar surfaces and interfaces, a variety of mechanisms such as reconstructions, defect formation, adsorption, and/or screening by free carriers in metallic substrates could take place to work against the electrostatic catastrophe, leading to rather complicated atomic structures depending 
on the temperature, atmosphere, and preparation conditions. The calculations for the (110) surfaces of the diamond and zinc-blende phases are conducted using 14 layer (18-39 ^) thick slabs separated by the same amount of vacuum and $6 \times 4 \times 1 k$ points. The (111) $2 \times 1$ reconstructed surface is also considered for $\mathrm{C}, \mathrm{Si}$, and $\mathrm{Ge}$ in the diamond structure, where 24 layer thick slabs and $6 \times 4 \times 1 k$ points are used. For the $(11 \overline{2} 0)$ and (1010) surfaces of wurtzite $\mathrm{ZnO}$ and $\mathrm{GaN}, 12$ and 14 layer-thick slabs, respectively, are used with $4 \times 4 \times 1$ and $6 \times 4 \times 1 k$ points, respectively. The ILPs are fixed to the PBEoptimized bulk values and the internal coordinates are relaxed. Subsequent PBE and HSE calculations are conducted without further atomic relaxation after scaling the lattice constants to the experimental values for the IP and EA evaluation.

Nonpolar (110) heterointerfaces between diamond and/or zinc-blende phases are modeled using supercells. Here 11 layers each of the two phases are stacked in the [110] direction, and $6 \times 4 \times 1 k$ points are used in the calculations. For the wurtzite $\mathrm{ZnO} / \mathrm{GaN}$ interface, two interface planes, (1120) and (1010), are considered. Supercells composed of 13 and 14 layers for each of the two constituent phases, corresponding to a thickness of $\sim 22 \AA$, are used with $4 \times 4 \times 1$ and $6 \times 4 \times 1 k$ points, respectively. First, interface calculations are conducted using PBE, where the ILPs are fixed to the average of the PBE lattice parameters of the two phases and the out-ofplane lattice parameter and internal coordinates are allowed to relax. Second, HSE calculations are carried out for the heterointerfaces without further atomic relaxation after scaling lattice parameters to those estimated from the HSE-optimized bulk values using the procedure described in Ref. [53]. The results of these interface calculations are used to obtain natural band offsets at experimental lattice constants in conjunction with the bulk and surface calculations, as described in Sec. II B.

\section{B. Derivation of band alignments}

The IP is a physical quantity providing the difference between the vacuum level and the VBM for a considered material, and the negative of the IP can be directly used for the valence band alignment. The conduction band is aligned using the negative of the EA, denoting the difference between the vacuum level and the CBM. We consider the vertical IP and EA, where the contributions of atomic relaxations accompanied by the electron removal and addition are excluded by definition. The vertical IP is calculated using the bulk-based definition, which excludes the contributions of occupied surface states above the VBM, via electrostatic alignment between the surface and the bulk [32] as

$$
\varepsilon_{\mathrm{IP}}=\Delta \varepsilon_{\mathrm{Vac}-\mathrm{Ref}}-\Delta \varepsilon_{\mathrm{VBM}-\mathrm{Ref}},
$$

where $\Delta \varepsilon_{\mathrm{Vac}-\mathrm{Ref}}$ is the energy difference between the electrostatic potential in the vacuum region, namely the vacuum level, and the reference level in a bulklike region of a surface supercell. The latter is determined by averaging the local KohnSham potential within PAW spheres over atomic sites located in a region far from the surface within a thickness of one-third of the slab. $\Delta \varepsilon_{\mathrm{VBM}-\text { Ref }}$ is the energy difference between the VBM and the reference level from bulk calculations. This contribution is evaluated up to the $G W \Gamma^{1}$ level; for $G W$ and $G W \Gamma^{1}$, QP shifts of the VBM are added to $\Delta \varepsilon_{\mathrm{VBM}-\mathrm{Ref}}$ obtained using PBE or HSE. For consistency, the second term using PBE or $G W_{0} @ \mathrm{PBE}$ is combined with the first term using PBE in Eq. (1). Similarly, the second term using HSE, $G W^{\mathrm{TC}-\mathrm{TC}} @ \mathrm{HSE}$, or $G W \Gamma^{1} @ \mathrm{HSE}$ is combined with the first term using HSE. The first term from the surface calculations does not include many-body corrections. The corrections could, in principle, be considered using self-consistent $G W$ with an orbital update for surfaces; however, such calculations are computationally too demanding for the supercells used in the present study. A previous QP self-consistent $G W @ \mathrm{GGA}$ calculation of a $\mathrm{Si} / \mathrm{SiO}_{2}$ interface shows that many-body corrections to the electrostatic potential change the band offset by only $\sim 20 \mathrm{meV}$ [58]. This indicates that the electrostatic potential is already well described using the GGA even at an interface between a covalent semiconductor and an ionic insulator.

The calculated IPs are not very sensitive to the lattice constants for all considered systems. Both PBE and HSE IP values change by less than $0.1 \mathrm{eV}$ when the lattice constants optimized using the respective functionals are used instead of the experimental values; EAs are more sensitive to the lattice constants. For wurtzite $\mathrm{GaN}$ and $\mathrm{ZnO}$, which have an internal coordinate $u$, the bulk calculations can be made using either PBE-optimized or experimental $u$ values in combination with experimental lattice constants. The resultant IP values, however, differ by only $0.01 \mathrm{eV}$. The EA is obtained by subtracting the calculated band gap from the IP (Table I).

The natural band offsets at heterointerfaces are evaluated using the definition given in Ref. [53]. Basically, the valence band offset can be obtained by adding the following contributions: (i) energy differences between the VBM and the reference level of the perfect bulk obtained for the two constituent phases and (ii) the difference of the reference levels of two phases in a heterointerface model. The average local potential at atomic sites in the bulk or a bulklike region far from the interface is used for the reference level in the same manner as for the IP evaluation. One issue is that lattice-matched interface calculations must include strain in the two constituent phases, whereas, to predict the natural band offsets, the bulk calculations should use unstrained lattices assuming complete relief of interfacial strain. In this study we determine the reference level offsets between these strained and unstrained lattices through alignment of the strained and unstrained surfaces using the vacuum level as a common reference $[53,74]$. The alignment between the strained and unstrained lattices could instead be made using deformation potentials, which are obtained by calculations of homointerfaces between strained and unstrained lattices $[13,75,76]$. However, this procedure is not adopted here.

In the present approach using surface calculations for the ILP corrections, the natural valence band offset is given as [53]

$$
\begin{aligned}
\Delta \varepsilon_{\text {VBM }}^{\mathrm{A}-\mathrm{B}} & \\
= & \left(\Delta \varepsilon_{\text {VBM-Ref,A }}^{\mathrm{A}}-\Delta \varepsilon_{\text {Vac-Ref,A }}^{\mathrm{A}}+\Delta \varepsilon_{\mathrm{Vac}-\operatorname{Ref}, \mathrm{X}}^{\mathrm{A}}\right)+\Delta \varepsilon_{\text {Ref, } \mathrm{X}}^{\mathrm{A}-\mathrm{B}} \\
& -\left(\Delta \varepsilon_{\mathrm{VBM}-\operatorname{Ref}, \mathrm{B}}^{\mathrm{B}}-\Delta \varepsilon_{\text {Vac-Ref,B }}^{\mathrm{B}}+\Delta \varepsilon_{\text {Vac-Ref,X }}^{\mathrm{B}}\right),
\end{aligned}
$$

where $\Delta \varepsilon_{\text {Ref,X }}^{\mathrm{A}-\mathrm{B}}$ is the difference in the reference levels of two phases $A$ and $B$ at ILP $X$ obtained via the calculation for a lattice-matched A/B interface. An average of the lattice 
constants of phases A and B is used for the ILP, as mentioned in Sec. II A. $\Delta \varepsilon_{\mathrm{VBM}-\text { Ref, } \mathrm{A}}^{\mathrm{A}}$ is the energy difference from the reference level to the VBM for bulk phase $A$ at its natural (unstrained) experimental lattice constant, which is determined using bulk calculations. Many-body corrections using $G W$ and $G W \Gamma^{1}$ are considered only for the bulk, as in the IP evaluation. $\Delta \varepsilon_{\text {Vac-Ref,A }}^{\mathrm{A}}\left(\Delta \varepsilon_{\mathrm{Vac}-\text { Ref, } \mathrm{X}}^{\mathrm{A}}\right)$ is the energy difference from a reference level near the center of the slab to the vacuum level for phase A with ILP A(X) obtained via surface calculations. $\Delta \varepsilon_{\mathrm{VBM}-\text { Ref,B }}^{\mathrm{B}}, \Delta \varepsilon_{\mathrm{Vac}-\text { Ref,B }}^{\mathrm{B}}$, and $\Delta \varepsilon_{\mathrm{Vac}-\text { Ref,X }}^{\mathrm{B}}$ denote similar quantities regarding phase $\mathrm{B}$. The $\mathrm{PBE}$ and $G W_{0} @ \mathrm{PBE}$ results or the HSE, $G W^{\mathrm{TC}-\mathrm{TC}} @ \mathrm{HSE}$, and $G W \Gamma^{1} @ \mathrm{HSE}$ results are consistently used for all terms in Eq. (2). The conduction band offset is obtained by adding the difference in the calculated band gaps of the two phases (Table I) to the valence band offset.

The evaluation of the natural band offset according to the definition in Eq. (2) requires surface calculations at the average ILPs, which are used in the coherent interface calculation, as well as the experimental ILPs. Hence, when considering many combinations of materials, omitting corrections for the change in the reference level arising from the change in the lattice parameters from the interface to the bulk would significantly reduce the number of calculations. This approximation is, however, only reasonable if the change in the reference level with ILPs is small. For instance, our previous studies found that such a simplified evaluation of natural band offsets yields the same values within $\sim 0.1 \mathrm{eV}$ as explicitly including the ILP changes in the $\mathrm{CuInSe}_{2} / \mathrm{ZnS}$ and $\mathrm{CdSnP}_{2} / \mathrm{ZnS}$ systems where the misfits in the ILPs are $\sim 7 \%$ and $\sim 10 \%$, respectively $[29,53]$. The reference levels as a function of the ILPs shown in Fig. 1, however, indicate that this is fortuitous because $\mathrm{ZnS}$ and CdS have exceedingly small dependencies of the reference levels on the ILPs and that the reference level changes could be sizable in many cases. We therefore consider the contributions from the ILP changes in Eq. (2) in the present study. With that being said, Fig. 1 also shows that this contribution is not significant for interfaces with a small or moderate lattice misfit, as expected. Even for Si that shows the largest change against the lattice parameter, the reference level change is $0.04 \mathrm{eV}$ for a $1 \%$ lattice misfit (a $0.5 \%$ ILP change from the interface with an average ILP to the bulk). The profile for Ge shows a relatively large fluctuation with the ILP, which is due to a transition in the surface atomic and electronic structure. This may lead to an uncertainty of $\sim 0.1 \mathrm{eV}$ for the interfacial band offsets involving $\mathrm{Ge}$.

The above procedure yields natural band offsets between any two materials, and we determine average band alignments using these interfacial band offsets. We consider 17 semiconductors in the diamond and zinc-blende structures, namely $\mathrm{Si}$, Ge, nine III-V semiconductors AlP, AlAs, AlSb, GaP, GaAs, $\mathrm{GaSb}$, InP, InAs, and InSb, and six II-VI semiconductors ZnS, $\mathrm{ZnSe}, \mathrm{ZnTe}, \mathrm{CdS}, \mathrm{CdSe}$, and CdTe, for the evaluation of this average offset-based band alignment. Among these systems, the maximum misfit is $20 \%$ for the combination of CdTe and $\mathrm{ZnS}$ (Table I). The average band alignment of phase A, $\varepsilon_{\mathrm{offset}}^{\mathrm{A}}$, is obtained by averaging the offsets over all $\mathrm{A} / \mathrm{B}$ interfaces as $\varepsilon_{\text {offset }}^{\mathrm{A}}=\sum_{\mathrm{B}} \Delta \varepsilon_{\mathrm{VBM}}^{\mathrm{A}-\mathrm{B}} / N$, where $N$ is the number of phases considered as $\mathrm{B}$. In other words, we define the interfacial band alignment to be the band offset against a hypothetical average of the VBM of the considered phases B. The absolute value of $\varepsilon_{\text {offset }}^{\mathrm{A}}$ has no physical meaning. Note that the set of phases considered as A must be exactly the same as the set of phases B considered as counterparts. A similar band alignment using average interfacial band offsets has been constructed, for instance, by $\mathrm{Li}$ et al. via LDA calculations [13]. C and BN, which have exceedingly small lattice constants and thereby huge misfits with the aforementioned substances, are treated separately, as well as $\mathrm{ZnO}$ and $\mathrm{GaN}$ in the wurtzite structure. The band offsets between 139 heterointerfaces are calculated, which include all 136 combinations between the 17 semiconductors, $\mathrm{C} / \mathrm{BN}$, and two types of $\mathrm{GaN} / \mathrm{ZnO}$ interfaces.

The branch point energy can be approximated as the average of high lying valence bands and low lying conduction bands [8-10,14,15]. We take the approach reported by Schleife $e t$ al. [15], with which the branch point energy $\varepsilon_{\mathrm{BP}}$ is given as

$$
\varepsilon_{\mathrm{BP}}=\frac{1}{2 N_{k}} \sum_{\mathbf{k}}\left[\frac{1}{N_{\mathrm{VB}}} \sum_{i}^{N_{\mathrm{VB}}} \varepsilon_{i}(\mathbf{k})+\frac{1}{N_{\mathrm{CB}}} \sum_{j}^{N_{\mathrm{CB}}} \varepsilon_{j}(\mathbf{k})\right] \text {, }
$$

where $N_{k}$ is the number of $k$ points in the Brillouin zone, $\varepsilon_{i}(\mathbf{k})$ denotes the $i$ th highest valence band at wave vector $\mathbf{k}$, which is sampled up to $i=N_{\mathrm{VB}}$, and $\varepsilon_{j}(\mathbf{k})$ is the $j$ th lowest conduction band at $\mathbf{k}$ and sampled up to $j=N_{\mathrm{CB}}$. The two highest valence
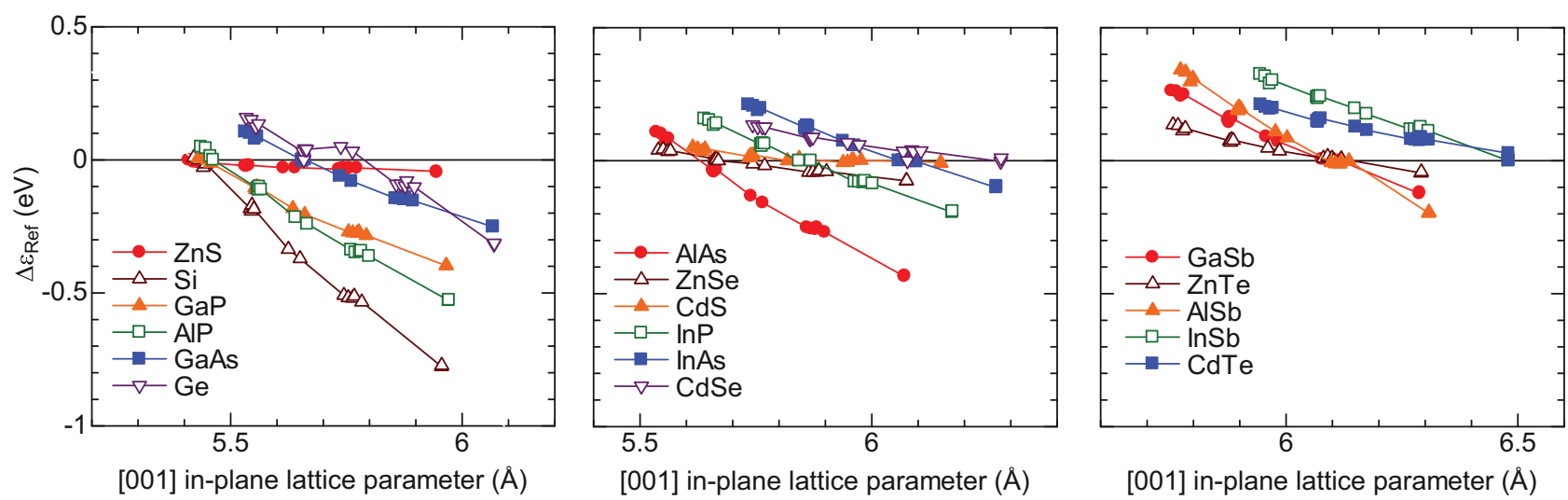

FIG. 1. (Color online) Average atomic-site local Kohn-Sham potential in a bulklike region against the vacuum level as a function of the in-plane lattice parameter in the [001] direction, obtained using HSE for zinc-blende (110) surface supercells. Values relative to that for the experimental lattice constant are shown for each material. 
bands and the lowest conduction band are considered for the diamond and zinc-blende phases $\left(N_{\mathrm{VB}}=2, N_{\mathrm{CB}}=1\right)$. The third valence band is not included here because it shows much larger dispersion than the first and second valence bands [15]. $N_{\mathrm{VB}}=4$ and $N_{\mathrm{CB}}=2$ are used for the wurtzite phases that contain twice as many atoms in the primitive cell as the zinc-blende phases. The branch-point energies obtained using Eq. (3) depend on the number of the sampled bands $\left(N_{\mathrm{VB}}\right.$ and $N_{\mathrm{CB}}$ ). However, we found that changing $N_{\mathrm{VB}}$ and $N_{\mathrm{CB}}$ values tends to cause uniform shifts in the branch point energies of the semiconductors considered here and hence the alignment is not substantially altered, as discussed in Sec. III C. The band average is taken over a $\Gamma$-centered $6 \times 6 \times 6 k$-point mesh in the primitive cell using the $G W^{\mathrm{TC}-\mathrm{TC}} \mathrm{QP}$ energies. The conduction bands are rigidly shifted so that the band gaps match the $G W \Gamma^{1}$ or experimental values (Table I), yielding two sets of branch point energies: The former is not evaluated solely using the $G W \Gamma^{1} \mathrm{QP}$ energies because the contributions from self-energy vertex corrections are calculated using a reduced $4 \times 4 \times 4 k$ point mesh. Note that the correction terms for the former, namely the differences in the band gaps between $G W \Gamma^{1}$ and $G W^{\mathrm{TC}-\mathrm{TC}}$, are small at $\sim 0.2 \mathrm{eV}$ for $\mathrm{BN}$ and $\mathrm{ZnO}$, and $\sim 0.1 \mathrm{eV}$ or less for the rest of the semiconductors considered in this study, as shown in Table I. Those for the latter, namely the differences between the experimental and $G W^{\text {TC-TC }}$ values, are $\sim 0.4 \mathrm{eV}$ for $\mathrm{C}$ and $\mathrm{BN}, \sim 0.3 \mathrm{eV}$ for $\mathrm{ZnO}$, and $\sim 0.2 \mathrm{eV}$ or less for the rest. These errors can be partly due to the effects of electron-phonon coupling as discussed later.

\section{RESULTS AND DISCUSSION}

\section{A. IP- and EA-based band alignment}

We begin with the IP- and EA-based band alignment for its rigorous and unambiguous definition. Figure 2 shows IP- and EA-based band alignments using $G W \Gamma^{1}$ compared with available experimental values [77,78]. Small differences in the IPs of Ge, and the Ga and In compounds from those reported in our previous study (up to $0.1 \mathrm{eV}$ ) [21] arise from the use of different computational settings. In the present calculations, PAW data sets excluding relatively deep Ga $3 d$, Ge $3 d$, and In $4 d$ states from the valence are consistently used for the surface, interface, and bulk calculations at the PBE and HSE levels, although all $G W$ type calculations apply the potentials summarized in the accompanying material of Ref. [21]. This reduces the computational costs, in particular, for the large number of interface calculations. The experimental EA values are obtained by subtracting the experimental band gaps (Table I) from the experimental IPs summarized in Ref. [77]; an exception is the $\mathrm{ZnO}$ (1010) surface, for which an experimental EA value has been reported [78] and the IP is estimated using the experimental EA and band gap. Among the approximations considered in this study, $G W \Gamma^{1}$ best reproduces the experimental IP and EA values as discussed below. The $G W \Gamma^{1}$ band gaps listed in Table I also agree well with experimental values in general, with an overestimation typically by $\sim 0.2 \mathrm{eV}$ or less. The errors can be partly attributed to the band gap reduction associated with the electron-phonon coupling, e.g., 0.1, 0.15, and $0.4 \mathrm{eV}$ for $\mathrm{Si}$ [79], $\mathrm{ZnO}$ [80], and $\mathrm{C}$ [81], respectively, and also to the lattice polarization contributions to the screening in polar materials [82,83], both of which are not accounted for in our theoretical treatment.

As the IP and EA are inevitably affected by surface dipoles, these quantities depend on the orientation, reconstruction, and adsorbate coverage of the surfaces. The experimental values for the surface orientations and reconstructed structures identical to those for the calculations are collected in Fig. 2 for comparison. An exception is $\mathrm{GaN}$, for which experimental IP and EA values for nonpolar surfaces are not available and

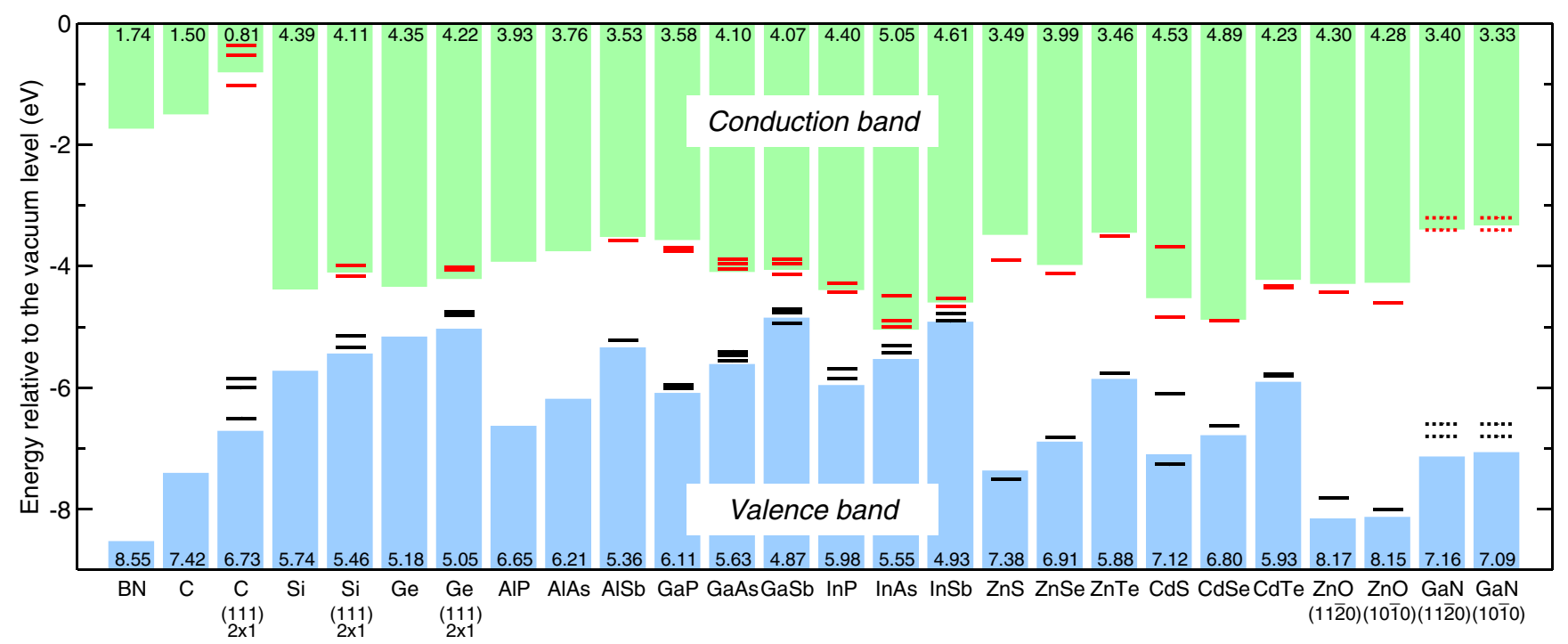

FIG. 2. (Color online) Band alignment based on the ionization potential (IP) and electron affinity (EA) obtained using $G W \Gamma^{1} @ H S E$. The surface orientation is (110) unless otherwise noted. Experimental values reported for the corresponding surfaces are indicated by solid horizontal bars except for GaN, for which values for polar $\{0001\}$ surfaces are shown with broken bars [77,78]. The theoretical IP and EA values are shown as numbers at the bottom and top of the figure, respectively. Note that the IP and EA correspond to the negatives of the valence band maximum and conduction band minimum with respect to the vacuum level, respectively. 
reported values for the $\{0001\}$ polar surfaces [77] are shown instead. Some experimental IPs show a spread when more than one value is reported for the same surface orientation. In particular, this is noticeable for $\mathrm{C}$, InAs, and $\mathrm{CdS}$, which may be partly due to adsorbates. Although the surface-dependent nature makes detailed comparison difficult for some systems, it is clear that $G W \Gamma^{1}$ generally reproduces the IPs and EAs well. The differences in the IP and EA between theory and experiment are on average $\sim 0.2 \mathrm{eV}$ but largely material dependent. This might be partly attributed to the contributions from electron-phonon coupling, as mentioned above.

Two kinds of nonpolar surface planes are considered for $\mathrm{ZnO}$ and $\mathrm{GaN}$ in the wurtzite structure and $\mathrm{C}, \mathrm{Si}$, and $\mathrm{Ge}$ in the diamond structure. The nonpolar (1120) and (10 $\overline{1} 0)$ surfaces show nearly the same IPs and EAs for both $\mathrm{ZnO}$ and $\mathrm{GaN}$. Similar behavior is found in the previously reported PBE results for the nonpolar surfaces of $\mathrm{GaN}$ as well as AlN and InN [51]. Surface energy calculations indicate that the unreconstructed (110) surfaces of $\mathrm{C}, \mathrm{Si}$, and $\mathrm{Ge}$ are energetically much less favorable than the experimentally well-characterized (111) $2 \times 1$ reconstructed surfaces. In addition, experimental IP values are not available for the unreconstructed (110) surfaces. Nevertheless, we include the results for the (110) surfaces to discuss the orientation dependence of the IP and EA. In contrast to the case of the wurtzite compounds, the IP and EA for the diamond-structure semiconductors have noticeable orientation dependencies. The (110) surfaces show consistently larger IP and EA (lower VBM and $\mathrm{CBM}$ ) values than the (111) $2 \times 1$ surfaces. A particularly large surface-dependence of $0.7 \mathrm{eV}$ is found for $\mathrm{C}$. The dependence is much smaller for $\mathrm{Si}$ and $\mathrm{Ge}$, with differences of 0.28 and $0.13 \mathrm{eV}$ between the (110) and (111) $2 \times 1$ surfaces, respectively.

Figure 3 compares PBE, HSE, $G W_{0} @ \mathrm{PBE}, G W^{\mathrm{TC}-\mathrm{TC}}$, and $G W \Gamma^{1}$ results for the VBM and CBM with respect to the vacuum level, and the band gap. The considered surfaces are (111) $2 \times 1$ for $\mathrm{C}, \mathrm{Si}$, and $\mathrm{Ge}$ in the diamond structure, (112̄0) for $\mathrm{GaN}$ and $\mathrm{ZnO}$ in the wurtzite structure, and (110) for the rest of the compounds in the zinc-blende structure. The differences between the $G W_{0} @ \mathrm{PBE}$ and PBE values correspond to the bulk QP shifts from PBE. Similarly, the differences between $G W^{\mathrm{TC}-\mathrm{TC}}$ and HSE are equal to the bulk QP shifts from HSE. The differences between the $G W \Gamma^{1}$ and $G W^{\mathrm{TC}-\mathrm{TC}}$ values indicate the contributions of the vertex corrections in the self-energy included in $G W \Gamma^{1}$ on top of $G W^{\mathrm{TC}-\mathrm{TC}}$. Note that the QP shifts in the IP and EA correspond to the negatives of the VBM and CBM shifts, respectively.

The PBE semilocal functional, which is a standard approximation to DFT, shows the largest deviation in the band gap and VBM from the experimental values. The band gaps are underestimated by $\sim 1 \mathrm{eV}$ on average. The $\mathrm{VBM}$ is too shallow with respect to the vacuum level by a similar amount. In other words, the IPs are too small for PBE. This tendency is especially evident for the $\mathrm{Zn}$ and $\mathrm{Cd}$ chalcogenides with wide band gaps. In contrast, the CBM is much better reproduced. For $\mathrm{ZnO}$, the CBM is too deep and therefore the EA is too large by $0.8 \mathrm{eV}$. The error, however, is smaller than that of the VBM or IP. HSE improves upon PBE for the VBMs and band gaps of most semiconductors. Still, it shows sizable differences from the experimental values for the group II-VI semiconductors.
The deviations in the CBM are smaller than those in the VBM as in the case of PBE.

As expected from the previous statement that $W_{0} @ \mathrm{PBE} \approx$ $W^{\mathrm{TC}-\mathrm{TC}} @ \mathrm{HSE}$ [73], $G W_{0} @ \mathrm{PBE}$ and $G W^{\mathrm{TC}-\mathrm{TC}}$ behave similarly for most semiconductors; the discrepancies in Ge and $\mathrm{ZnO}$ are partly related to the significant underestimation of the band gaps by $\mathrm{PBE}$ and a resultant inaccurate $W_{0}$. These approximations yield VBMs and CBMs lower than the experimental values for almost all the considered semiconductors. $G W \Gamma^{1}$ gives clear improvements on this point. $G W^{\text {TC-TC }}$ shows nearly constant differences from $G W \Gamma^{1}$, with average deviations of 0.2 and $0.3 \mathrm{eV}$ in the VBM, or IP, and CBM, or EA, respectively. A constant shift will result in a sizable error cancellation, when relative $\mathrm{VBM}$ and CBM values are investigated between different materials. The additional contribution of $G W \Gamma^{1}$ on top of $G W^{\mathrm{TC}-\mathrm{TC}}$, which is associated with the vertex corrections in the self-energy, shifts the extended states in the valence and conduction bands upwards towards the vacuum level typically by 0.2 and $0.3 \mathrm{eV}$, respectively, for the group IV, III-V, and II-VI semiconductors; exceptions are the valence states of oxides including $\mathrm{ZnO}$, which hardly shift because of their relatively localized character [21]. Therefore, the band gaps of $G W^{\mathrm{TC}-\mathrm{TC}}$ and $G W \Gamma^{1}$ are generally close to each other as recognized in Fig. 3 and Table I.

The QP shifts of the VBM are generally larger than those of the CBM for all $G W$ flavors. This might be in contrast to the common conjecture that the valence band positions are well described by DFT. The main reason for our relatively large VBM QP shifts is that we carefully converged the QP corrections with respect to the basis sets. Exceedingly slow convergence has been first observed in recent studies on $\mathrm{ZnO}[72,84]$ and we observe similar issues for all materials considered here, in particular for the absolute QP shifts. In the present study we have carefully estimated the QP shifts at the infinite basis set limit via extrapolation for all the considered materials [21,71,72]. Furthermore, we used approximately norm-conserving PAW potentials. These have been shown to be crucial in order to obtain highly accurate results [21,71]. We note that a recent $G_{0} W_{0} @$ PBE study using a different code also shows the tendency that the VBM shifts are larger than the CBM shifts for some of the semiconductors considered in this study $[85,86]$.

In summary, PBE provides too high VBMs, or underestimates IPs, while the CBMs, or EAs, are reproduced much better for most semiconductors. HSE tends to overestimate both VBMs and CBMs (underestimate the IPs and EAs) but shows remarkable improvement in the VBM from PBE, as well as in the band gap. $G W_{0} @ \mathrm{PBE}$ and $G W^{\mathrm{TC}-\mathrm{TC}}$ slightly underestimate the VBMs and CBMs (overestimate the IPs and EAs). $G W \Gamma^{1}$ improves this and yields the overall best description. If we focus on the relative band positions between semiconductors, for example to predict interfacial band offsets, error cancellation is expected. In other words, the differences in the VBM and CBM with respect to the vacuum level between two materials ( $\triangle \mathrm{VBM}$ and $\triangle \mathrm{CBM}$, respectively) using $\mathrm{PBE}$, HSE, $G W_{0} @ \mathrm{PBE}$, and $G W^{\mathrm{TC}-\mathrm{TC}}$ agree much better with the $G W \Gamma^{1}$ results than the absolute values. For instance, if we use $\mathrm{Si}$ as a reference, errors in $\triangle \mathrm{CBM}$ against $G W \Gamma^{1}$ are $-0.23,0.05,-0.11$, and $0.08 \mathrm{eV}$ on average for PBE, HSE, 

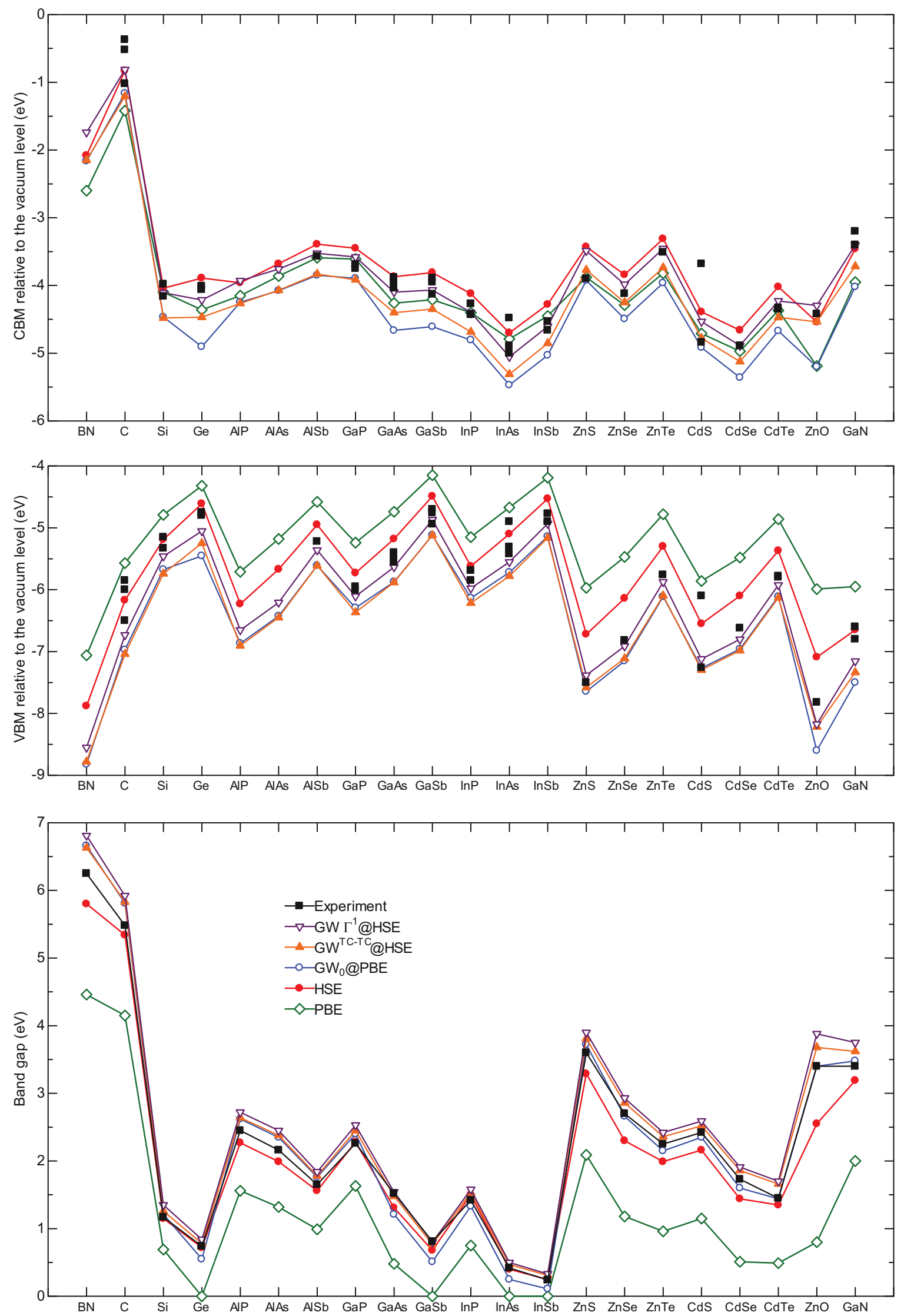

FIG. 3. (Color online) Conduction band minima (CBMs) relative to the vacuum level (negative of the electron affinities), valence band maxima (VBMs) relative to the vacuum level (negative of the ionization potentials), and the band gaps obtained using various approximations along with reported experimental values [77]. The theoretical and experimental values for the band gaps are listed in Table I. 
TABLE II. Theoretical natural valence band offsets for $\mathrm{A} / \mathrm{B}$ heterointerfaces obtained using various approximations along with experimental values. Positive values mean that the valence band maximum of semiconductor $\mathrm{A}$ is higher than that of semiconductor $\mathrm{B}$. The theoretical values are for diamond and zinc-blende (110) interfaces and the GaN/ZnO (1010) and (1120) interfaces (the latter in parentheses). The experimental values are not necessarily for natural (unstrained) band offsets (see text).

\begin{tabular}{|c|c|c|c|c|c|c|c|c|c|}
\hline \multirow[b]{2}{*}{ Interface $(\mathrm{A} / \mathrm{B})$} & \multirow[b]{2}{*}{ Misfit } & \multicolumn{5}{|c|}{ Theoretical offset (eV) } & \multicolumn{3}{|c|}{ Experiment } \\
\hline & & PBE & HSE & $G W_{0} @ \mathrm{PBE}$ & $G W^{\mathrm{TC}-\mathrm{TC}} @ \mathrm{HSE}$ & $G W \Gamma^{1} @ \mathrm{HSE}$ & Offset $(\mathrm{eV})$ & Interface orientation & Ref. \\
\hline \multirow[t]{2}{*}{$\mathrm{GaP} / \mathrm{AlP}$} & $0.2 \%$ & 0.46 & 0.53 & 0.56 & 0.57 & 0.58 & 0.57 & GaP/AlP/GaP (100) & [97] \\
\hline & & & & & & & 0.43 & $\mathrm{AlP}$ on $\mathrm{GaP}(100)$ & [98] \\
\hline $\mathrm{Si} / \mathrm{GaP}$ & $0.4 \%$ & 0.32 & 0.40 & 0.49 & 0.49 & 0.51 & 0.80 & Si on GaP (110) & [99] \\
\hline \multirow[t]{4}{*}{ GaAs/AlAs } & $0.1 \%$ & 0.45 & 0.47 & 0.56 & 0.55 & 0.55 & 0.40 & GaAs on AlAs (110) & [100] \\
\hline & & & & & & & 0.15 & AlAs on GaAs (110) & [100] \\
\hline & & & & & & & 0.55 & AlAs on GaAs (100) & [101] \\
\hline & & & & & & & $0.45 \pm 0.05$ & AlAs on GaAs (100) & [102] \\
\hline \multirow[t]{3}{*}{$\mathrm{GaSb} / \mathrm{AlSb}$} & $0.6 \%$ & 0.42 & 0.45 & 0.48 & 0.48 & 0.48 & $0.40 \pm 0.15$ & $\mathrm{AlSb}$ on GaSb (100) & [103] \\
\hline & & & & & & & $0.39 \pm 0.07$ & AlSb on GaSb (100) & [104] \\
\hline & & & & & & & $0.40 \pm 0.15$ & GaSb on AlSb (100) & [103] \\
\hline \multirow{2}{*}{$\mathrm{ZnTe} / \mathrm{AlSb}$} & $0.5 \%$ & -0.24 & -0.42 & -0.55 & -0.56 & -0.59 & $-0.42 \pm 0.07$ & AlSb on ZnTe (100) & [104] \\
\hline & & & & & & & $-0.35 \pm 0.11$ & AlSb on ZnTe (100) & [105] \\
\hline \multirow[t]{2}{*}{ Ge/AlAs } & $0.1 \%$ & 0.95 & 1.10 & 1.06 & 1.24 & 1.19 & $0.95 \pm 0.2$ & Ge on AlAs (110) & [106] \\
\hline & & & & & & & 0.9 & $(110)$ & [107] \\
\hline \multirow[t]{3}{*}{$\mathrm{GaAs} / \mathrm{Ge}$} & $0.1 \%$ & -0.31 & -0.55 & -0.31 & -0.61 & -0.56 & $\begin{array}{l}-0.54 \pm 0.05 \text { to } \\
-0.59 \pm 0.05\end{array}$ & Ge on GaAs (110) & [108] \\
\hline & & & & & & & -0.42 & Ge on GaAs (110) & [109] \\
\hline & & & & & & & $-0.53 \pm 0.03$ & $(110)$ & [110] \\
\hline $\mathrm{InP} / \mathrm{InAs}$ & $3.2 \%$ & -0.25 & -0.44 & -0.18 & -0.36 & -0.35 & -0.31 & $(100)$ & [111] \\
\hline GaAs/InP & $3.8 \%$ & 0.44 & 0.53 & 0.29 & 0.42 & 0.43 & 0.19 & $(100)$ & [111] \\
\hline \multirow[t]{2}{*}{$\mathrm{Ge} / \mathrm{ZnSe}$} & $0.2 \%$ & 0.96 & 1.52 & 1.51 & 1.85 & 1.85 & 1.52 & Ge on $\mathrm{ZnSe}(110)$ & [112] \\
\hline & & & & & & & 1.29 & $\mathrm{ZnSe}$ on $\mathrm{Ge}(110)$ & [112] \\
\hline $\mathrm{CdSe} / \mathrm{ZnTe}$ & $0.4 \%$ & -0.62 & -0.79 & -0.77 & -0.88 & -0.92 & $-0.64 \pm 0.07$ & $(100)$ & [113] \\
\hline $\mathrm{GaAs} / \mathrm{ZnSe}$ & $0.3 \%$ & 0.65 & 0.93 & 1.20 & 1.20 & 1.25 & 0.96 to 1.10 & ZnSe on GaAs (110) & [112] \\
\hline \multirow[t]{2}{*}{$\mathrm{GaSb} / \mathrm{ZnTe}$} & $0.1 \%$ & 0.54 & 0.77 & 0.90 & 0.94 & 0.96 & 0.34 & $\mathrm{ZnTe}$ on GaSb (110) & [114] \\
\hline & & & & & & & $0.60 \pm 0.07$ & ZnTe on GaSb (100) & [104] \\
\hline $\mathrm{InSb} / \mathrm{CdTe}$ & $0.0 \%$ & 0.39 & 0.70 & 0.69 & 0.83 & 0.86 & $0.87 \pm 0.10$ & CdTe on InSb & [115] \\
\hline \multirow[t]{3}{*}{$\mathrm{GaN} / \mathrm{ZnO}$} & $1.1 \%$ & 0.20 & 0.74 & 1.26 & 1.18 & 1.32 & $0.9 \pm 0.1$ & $\mathrm{GaN}$ on $\mathrm{ZnO}(10 \overline{1} 0)$ & [87] \\
\hline & & $(0.32)$ & $(0.79)$ & $(1.38)$ & $(1.23)$ & $(1.37)$ & $0.7 \pm 0.1$ & $\mathrm{GaN}$ on $\mathrm{ZnO}(0001)$ & {$[87]$} \\
\hline & & & & & & & $0.8,1.0$ & $\mathrm{ZnO}$ on $\mathrm{GaN}(0001)$ & {$[88]$} \\
\hline
\end{tabular}

$G W_{0} @ \mathrm{PBE}$, and $G W^{\mathrm{TC}-\mathrm{TC}}$, respectively. For $\Delta \mathrm{VBM}$ with respect to $\mathrm{Si}$, the average errors against $G W \Gamma^{1}$ are 0.41 , $0.26,-0.02$, and $0.06 \mathrm{eV}$, respectively. For relative values, $G W_{0} @ \mathrm{PBE}$ and $G W^{\mathrm{TC}-\mathrm{TC}}$ are thus on par with $G W \Gamma^{1}$ for most semiconductors. PBE and HSE perform reasonably well for $\triangle \mathrm{VBM}$ and $\triangle \mathrm{CBM}$, but we should note that the maximum errors in $\triangle \mathrm{VBM}$ can be as large as 1.5 and $0.8 \mathrm{eV}$, respectively.

\section{B. Interfacial offset-based band alignment}

Table II shows theoretical natural valence band offsets obtained using various approximations and experimental values for interfaces with relatively small lattice misfits. The offset values calculated using $G W \Gamma^{1}$ are listed for all the pairs of the 17 semiconductors in Table III. We also include experimental values for interface orientations other than diamond and zinc-blende (110) and wurtzite (1120) and (1010) that are considered in the interface calculations in Table II. A comparison between different interfaces is still meaningful, provided the interfacial orientation dependence is small.
The band offsets obtained using $G W \Gamma^{1}$ are close to previously reported values using $G W @$ LDA with a generalized plasmon-pole model [57], $G_{0} W_{0} @ \mathrm{PBE}$ with a Godby-Needs plasmon-pole model [26], and as well as our $G W_{0} @ \mathrm{PBE}$ and $G W^{\mathrm{TC}-\mathrm{TC}}$ values for most interfaces. In addition, the differences from the reported LDA [7] and HSE06 [54] values, and our PBE and HSE values are not significant. This is attributed partly to error cancellation in the evaluation of valence band offsets that require only relative valence band positions, as mentioned in Sec. III A for the IPs and EAs. Similar discussion is given afterwards regarding band offsets. Relatively large differences between $G W_{0} @ \mathrm{PBE}$ and $G W^{\mathrm{TC}-\mathrm{TC}}$ values are found for the interfaces involving $\mathrm{Ge}$, InAs, and InSb. This is partly attributed to their negligible band gaps with PBE in the bulk and/or the interface supercells, which lead to errors in the $G W_{0} @$ PBE offsets.

Agreement in the band offsets between $G W \Gamma^{1}$ and experiment is generally good, although for some systems the experimental values show a spread and the comparison is not straightforward. A relatively large difference between the experimental and $G W \Gamma^{1}$ offsets is found for GaN/ZnO. 
TABLE III. Natural valence band offsets for A/B heterointerfaces obtained using $G W \Gamma^{1} @ \mathrm{HSE}$ for pairs of 17 diamond and zinc-blende semiconductors (in eV). Positive values mean that the valence band maximum of semiconductor A is higher than that of semiconductor $\mathrm{B}$.

\begin{tabular}{|c|c|c|c|c|c|c|c|c|c|c|c|c|c|c|c|c|c|}
\hline $\mathrm{A} / \mathrm{B}$ & $\mathrm{Si}$ & $\mathrm{Ge}$ & AlP & AlAs & $\mathrm{AlSb}$ & $\mathrm{GaP}$ & GaAs & $\mathrm{GaSb}$ & InP & InAs & $\mathrm{InSb}$ & $\mathrm{ZnS}$ & $\mathrm{ZnSe}$ & $\mathrm{ZnTe}$ & $\mathrm{CdS}$ & $\mathrm{CdSe}$ & $\mathrm{CdTe}$ \\
\hline & - & 0.3 & & & .26 & & 9 & 0. & & & & & & & 7 & 1.53 & 0.7 \\
\hline $\mathrm{Se}$ & 0.38 & - & 1 & & 0.49 & 8 & 56 & -0.01 & 0.85 & I & 55 & & & & 1 & .72 & 0.99 \\
\hline IP & -1.24 & -1.56 & - & -0.40 & -1.11 & -0.58 & 0.94 & -1.60 & -0.43 & -0.79 & -1.35 & 0.93 & 0.41 & & 0.81 & 0.44 & -0.42 \\
\hline 1As & -0.88 & -1.19 & 0.40 & - & -0.73 & -0.20 & -0.55 & -1.20 & -0.07 & -0.43 & -0.91 & 1.36 & 0.84 & -0.16 & 1.20 & 0.82 & -0.04 \\
\hline $1 \mathrm{Sb}$ & -0.26 & -0.49 & & 0.73 & - & 0.50 & 0 & -0.48 & & 0 & 28 & & & 0.59 & & 56 & 0.68 \\
\hline $\mathrm{aP}$ & -0.51 & -0.88 & 0.5 & 0.20 & -0.50 & - & -0.35 & -0.97 & 0.11 & -0.26 & -0.55 & 1.33 & 0.86 & -0.05 & & 5 & 0.00 \\
\hline aAs & -0.19 & -0.56 & 0.94 & 0.55 & -0.17 & 0.35 & - & -0.58 & 0.43 & 0.07 & -0.26 & 1.72 & 1.25 & 0.31 & 1 & 1.22 & 0.35 \\
\hline $\mathrm{aSb}$ & 0.14 & 0.01 & 1 & 1.20 & 0.48 & 0.97 & 0.58 & - & 1.07 & 0.72 & 0.18 & 2.44 & 1.98 & 0.96 & 2.27 & 1.90 & 1.05 \\
\hline $\mathrm{InP}$ & -0.64 & -0.85 & 0.43 & 0.07 & -0.61 & -0.11 & -0.43 & -1.07 & - & -0.35 & -0.60 & 1.19 & 0.74 & -0.16 & 1.03 & 0.72 & -0.11 \\
\hline As & -0.33 & -0.57 & 0.7 & 0.43 & -0.30 & 0.26 & -0.07 & -0.72 & 0.3 & - & -0.34 & 1.62 & 1. & 0.20 & 1. & 1.10 & 0.27 \\
\hline $\mathrm{InSb}$ & -0.04 & -0.55 & 1.35 & 0.91 & 0.28 & 0.55 & 0.26 & -0.18 & 0.60 & 0.34 & - & 1.99 & 1.44 & 0.76 & 1.71 & 1.50 & 0.86 \\
\hline $\mathrm{ZnS}$ & -2.04 & -2.30 & -0.93 & -1.36 & -2.22 & -1.33 & -1.72 & -2.44 & -1.19 & -1.62 & -1.99 & - & -0.53 & -1.49 & -0.13 & -0.49 & -1.43 \\
\hline $\mathrm{ZnSe}$ & -1.55 & -1.85 & -0.41 & -0.84 & -1.62 & -0.86 & -1.25 & -1.98 & -0.74 & -1.15 & -1.44 & 0.53 & - & -0.97 & 0.35 & -0.01 & -0.87 \\
\hline $\mathrm{ZnTe}$ & -0.67 & -0.89 & 0.57 & 0.16 & -0.59 & 0.05 & -0.31 & -0.96 & 0.16 & -0.20 & -0.76 & 1.49 & 0.97 & - & 1.30 & 0.92 & 0.11 \\
\hline $\mathrm{dS}$ & -1.87 & -2.01 & -0.81 & -1.20 & -2.03 & -1.17 & -1.52 & -2.27 & -1.03 & -1.43 & -1.71 & 0.13 & -0.35 & -1.30 & - & -0.34 & -1.24 \\
\hline $\mathrm{CdSe}$ & -1.53 & -1.72 & -0.44 & -0.82 & -1.56 & -0.85 & -1.22 & -1.90 & -0.72 & -1.10 & -1.50 & 0.49 & 0.01 & -0.92 & 0.34 & - & -0.83 \\
\hline $\mathrm{CdTe}$ & -0.79 & -0.99 & 0.42 & 0.04 & -0.68 & 0.00 & -0.35 & -1.05 & 0.11 & -0.27 & -0.86 & 1.43 & 0.87 & -0.11 & 1.24 & 0.83 & - \\
\hline
\end{tabular}

Valence band offsets of $0.7-1.0$ and $0.9 \pm 0.1 \mathrm{eV}$ have been obtained for polar (0001) and nonpolar (1010) GaN/ZnO interfaces, respectively, using ultraviolet and $\mathrm{x}$-ray photoemission spectroscopy $[87,88]$. A larger value of $1.37 \mathrm{eV}$ has also been reported using an $\mathrm{x}$-ray photoemission spectroscopy measurement of a $\mathrm{ZnO} / \mathrm{AlN}$ interface and assumption of transitivity among $\mathrm{ZnO} / \mathrm{AlN}$ and $\mathrm{AlN} / \mathrm{GaN}$ interfaces [89]. The resultant band alignment is consistently type II, where the VBM and CBM of $\mathrm{ZnO}$ are lower than those of $\mathrm{GaN}$, respectively. Our calculations support the type II band alignment, as shown in Fig. 4, and indicate that the difference between the two kinds of nonpolar surface orientations is small at $\sim 0.1 \mathrm{eV}$. The calculated offset values using $G W_{0} @$ PBE, $G W^{\mathrm{TC}-\mathrm{TC}}$, and $G W \Gamma^{1}$, however, are $0.3-0.4 \mathrm{eV}$ larger than the experimental value reported for the $\mathrm{GaN} / \mathrm{ZnO}(10 \overline{1} 0)$ interface [87].

There are several possible sources for the discrepancies between theory and experiment. Given the agreement in the theoretical and experimental values for the IPs and EAs as shown in Fig. 2, we expect that our $G W \Gamma^{1}$ calculations can also predict interfacial band offsets rather accurately. Renormalization associated with electron-phonon coupling can be a cause of residual errors as mentioned in Sec. III A. Furthermore, the theoretical natural valence band offsets shown in Table II are for unstrained interfaces, whereas the experimental interfaces are not necessarily unstrained. For instance, a large difference in the experimental valence band offset has been reported between Ge grown on $\mathrm{Si}$ (100) and

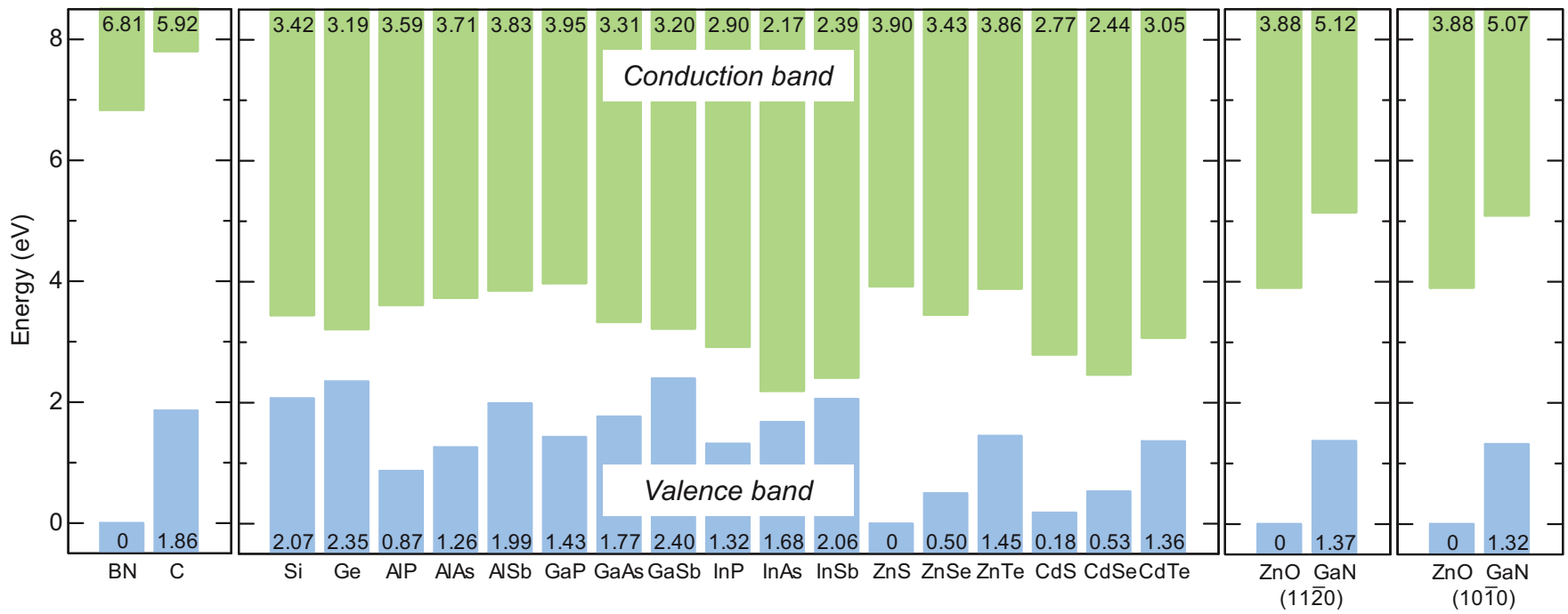

FIG. 4. (Color online) Band alignment based on the natural interfacial band offsets obtained using $G W \Gamma^{1} @$ HSE. The valence band maxima of $\mathrm{BN}, \mathrm{ZnS}$, and $\mathrm{ZnO}$ are set to zero in the respective panels. The alignment in the middle panel is constructed using average offset values; the natural valence band offsets for all pairs from the 17 semiconductors are listed in Table III. The relative values for the valence band maxima and conduction band minima in respective panels are shown as numbers at the bottom and top of the figure, respectively. 


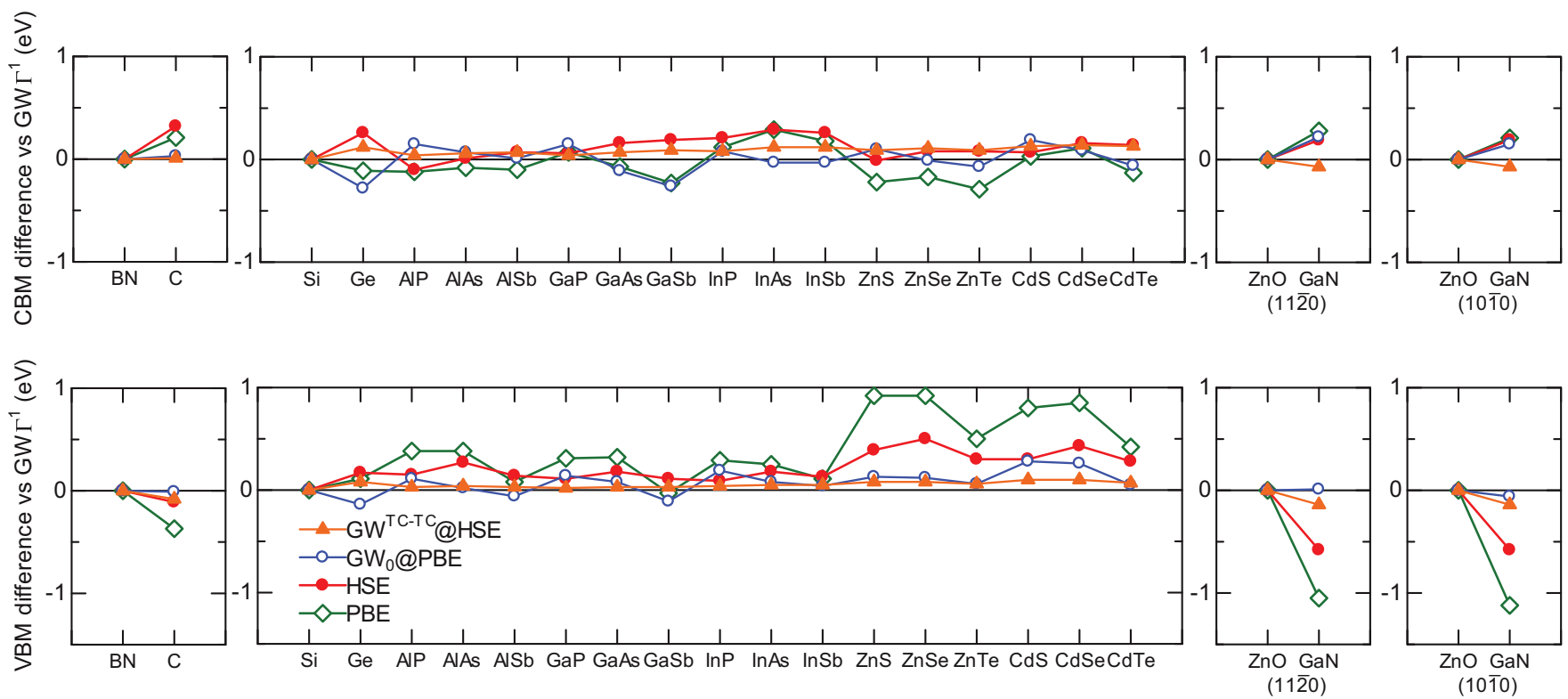

FIG. 5. (Color online) Deviations in the valence band maximum (VBM) and conduction band minimum (CBM) from $G W \Gamma^{1} @ H S E$ for PBE, HSE, $G W_{0} @ \mathrm{PBE}$, and $G W^{\mathrm{TC}-\mathrm{TC}} @ \mathrm{HSE}$ in the interfacial offset-based alignment. The values are relative to those for BN, Si, and ZnO in the respective panels.

Si grown on Ge (100), $-0.83 \pm 0.11$ and $-0.22 \pm 0.13 \mathrm{eV}$, respectively [90]. This has been attributed to strain effects [90]. Our natural (unstrained) band offset calculation using $G W \Gamma^{1}$ yields a value of $-0.38 \mathrm{eV}$ (Table III). The experimental offset values of other interfaces could also be affected by the strain although interfaces with relatively small lattice misfits are collected in Table II. For the interfacial atomic structure, we consider ideal, atomically flat interfaces in our calculations. However, actual interfaces may show reconstruction at the atomic level or include misfit dislocations to relieve the strain $[27,91,92]$. In addition, interdiffusion could occur across the interfaces [25,93]. All these could affect the interfacial dipoles. Our previous study for $\mathrm{CdTe} / \mathrm{CdS}, \mathrm{CdS} / \mathrm{ZnS}$, and $\mathrm{InP} / \mathrm{GaP}$ (110) interfaces indicates that an explicit consideration of misfit dislocations in the interface supercells changes the local potential typically by only $0.1 \mathrm{eV}$ or less at a distance of $\sim 1 \mathrm{~nm}$ from the dislocations [24]. Therefore, the effects of misfit dislocations on macroscopic band offsets are likely to be small. In other words, the natural band offsets obtained in this study would be good estimates above the critical thicknesses of interface constituents. A more likely source of the discrepancies between theory and experiment in Table II is, therefore, the reconstruction and interdiffusion as well as the strain.

The middle panel of Fig. 4 shows the band alignment derived from average interfacial band offsets for 17 semiconductors in the diamond and zinc-blende structures. The relative VBM and CBM are obtained from the data for the 136 heterointerfaces composed of the 17 semiconductors (Table III) using the procedure described in Sec. II B. The alignment reproduces actual $G W \Gamma^{1}$ band offsets with a mean absolute error of $0.06 \mathrm{eV}$ and a maximum deviation of $0.25 \mathrm{eV}$. The directly calculated offsets are shown in Fig. 4 for the BN/C interface as well as the $\mathrm{GaN} / \mathrm{ZnO}$ interfaces discussed above. A type II band alignment is predicted for the BN/C (110) interface, consistent with the IP-based band alignment in Fig. 2. The valence and conduction band offsets are as large as
1.8 and $0.9 \mathrm{eV}$, respectively. The type II alignment is unlikely to be altered even when the band gap reduction due to the phonon renormalization is considered: $\sim 0.4 \mathrm{eV}$ for $\mathrm{C}[81]$ while the value for $\mathrm{BN}$ has not been reported.

Offset-based band alignments are also constructed using the remaining four approximations and the deviations in the relative VBM and $\mathrm{CBM}$ from the $G W \Gamma^{1}$ results are shown in Fig. 5. The differences in band alignments are shown with respect to that of $\mathrm{Si}$ for the 17 semiconductors because of relatively small deviations of Si in the IP and EA from $G W \Gamma^{1}$ for all considered approximations, namely PBE, HSE, $G W_{0} @ \mathrm{PBE}$, and $G W^{\mathrm{TC}-\mathrm{TC}}$ (see Fig. 3). Since only the relative band positions are relevant for the band offsets, error cancellation is expected when errors in the VBM or CBM are roughly constant. Indeed, all approximations give values close to the $G W \Gamma^{1}$ values (which are set to zero in Fig. 5) for Ge and most of the III-V semiconductors. However, large deviations are found for the zinc-blende II-VI semiconductors when PBE is used. HSE improves the values, but they are still different by up to $0.5 \mathrm{eV}$ for the relative VBM of the zinc-blende II-VI semiconductors and $0.6 \mathrm{eV}$ for the wurtzite $\mathrm{ZnO} / \mathrm{GaN}$ interface. $G W_{0} @ \mathrm{PBE}$ yields values within $0.2 \mathrm{eV}$ with respect to $G W \Gamma^{1}$ for most systems, and behaves much better than HSE in selenides, sulfides, and $\mathrm{ZnO}$. $G W^{\mathrm{TC}-\mathrm{TC}}$ gives results very close to $G W \Gamma^{1}$ in all semiconductors. This is because the additional contribution of $G W \Gamma^{1}$ on top of $G W^{\mathrm{TC}-\mathrm{TC}}$, which is associated with the vertex correction in the self-energy, is consistently $0.2-0.3 \mathrm{eV}$ for both VBM and $\mathrm{CBM}$ of most semiconductors considered here [21], as already mentioned above.

\section{Prediction of interfacial band offsets using three approximate approaches}

The IP- and EA-based alignment is directly relevant if we need to predict the surface band positions, for instance, 

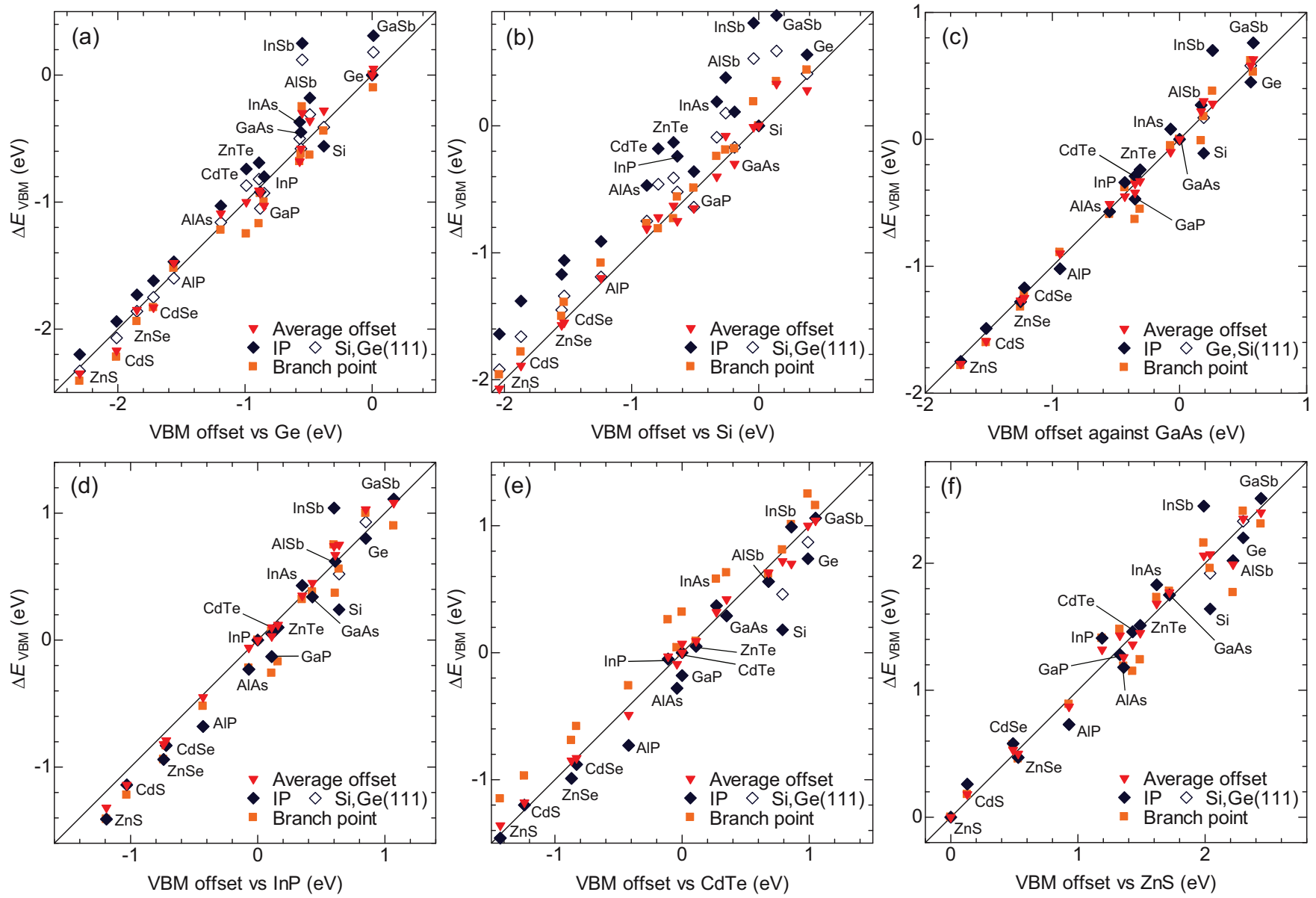

FIG. 6. (Color online) Valence band alignments based on average interfacial band offsets, ionization potentials (IPs), and branch point energies against interfacial band offsets explicitly calculated using $G W \Gamma^{1} @ \mathrm{HSE}$. The predicted valence band maxima (VBMs) relative to those of (a) Ge, (b) Si, (c) GaAs, (d) InP, (e) CdTe, and (f) $\mathrm{ZnS}$ are shown. Two sets of IP values are used for the IP-based alignment: one from (110) surfaces, and the other from (111) $2 \times 1$ surfaces for Si and Ge and (110) surfaces for the rest of the compounds.

to screen photocatalyst materials. Meanwhile, to predict interfacial band offsets in electronic devices and photovoltaic cells, a single band alignment diagram assuming transitivity would be very useful because systematic offset evaluation for a large number of interfaces are computationally demanding. In addition to the average interfacial offset-based and branch point energy-based alignments, the IP- and EA-based alignment can be used for this purpose, if the difference in the dipole contributions at two surfaces well approximates the contribution of the interfacial dipoles. The predictive power of these three transitive band alignments matters and needs to be inspected.

Figure 6 shows how well these band alignments can reproduce the directly calculated valence band offsets for interfaces involving $\mathrm{Ge}, \mathrm{Si}, \mathrm{GaAs}, \mathrm{InP}, \mathrm{CdTe}$, and $\mathrm{ZnS}$ using the $G W \Gamma^{1}$ results. The difference between the average interfacial offset-, IP-, and branch point energy-based alignments does not change between valence and conduction band alignments because the conduction band alignments differ constantly by the band gap of each semiconductor from the valence band alignments. Therefore, it suffices to inspect the VBM offsets.

The average interfacial offsets are distributed equally above and below the diagonal line in each panel by definition.
Considering the 136 combinations of the 17 semiconductors, the average interfacial offset-based alignments reproduce the actual offsets best, with a mean absolute error of $0.06 \mathrm{eV}$ and the maximum deviation of $0.25 \mathrm{eV}$ as mentioned above.

The alignment based on branch point energies shows relatively large errors for the interfaces involving $\mathrm{ZnTe}$ and CdTe. This is most obvious in Fig. 6(e) where offsets against CdTe are systematically overestimated by $\sim 0.2 \mathrm{eV}$ for most of the semiconductors. Agreement with the directly calculated interfacial offsets is generally good, with a mean absolute error of $0.14 \mathrm{eV}$ and a maximum deviation of $0.44 \mathrm{eV}$. Branch point energy-based alignment is also made for $\mathrm{BN} / \mathrm{C}$ and $\mathrm{GaN} / \mathrm{ZnO}$, yielding differences from the directly calculated valence band offsets of -0.32 and $-0.28 \mathrm{eV}$, respectively.

For future reference, the calculated branch point energies are listed in Table IV. Two sets of values were obtained: the $G W^{\mathrm{TC}-\mathrm{TC}}$ conduction band is shifted to match either the $G W \Gamma^{1}$ or experimental band gap. The former is used for the comparison with the other band alignments derived using $G W \Gamma^{1}$ in Fig. 6 and the discussion above. The latter is more suitable for comparison with existing literature, specifically, reported values from empirical tight-binding calculations [14] and linearized augmented plane-wave calculations using the 
TABLE IV. Branch point energies with respect to the valence band maxima obtained using $G W^{\mathrm{TC}-\mathrm{TC}} @$ HSE with a shift of the conduction bands to match the $G W \Gamma^{1} @ \operatorname{HSE}$ band gaps $\left(E_{\mathrm{g}}=E_{\mathrm{g}}^{\text {theory }}\right)$ or experimental band gaps $\left(E_{\mathrm{g}}=E_{\mathrm{g}}^{\text {expt }}\right)$. Reported values from empirical tight-binding calculations (ETB) [14] and linearized augmented plane-wave calculations with the LDA (LAPW-LDA) [94-96] are also shown as summarized in Ref. [14]. Values are in $\mathrm{eV}$.

\begin{tabular}{lrrrr}
\hline \hline & \multicolumn{2}{c}{ This work } & & \\
\cline { 2 - 3 } & $E_{\mathrm{g}}=E_{\mathrm{g}}^{\text {theory }}$ & $E_{\mathrm{g}}=E_{\mathrm{g}}^{\text {expt }}$ & & \\
ETB [14] & LAPW-LDA [94-96] \\
\hline $\mathrm{BN}$ & 3.39 & 3.11 & 3.25 & \\
$\mathrm{C}$ & 1.85 & 1.63 & 1.77 & \\
$\mathrm{Si}$ & 0.16 & 0.07 & 0.03 & 0.36 \\
$\mathrm{Ge}$ & -0.28 & -0.32 & -0.28 & 0.18 \\
$\mathrm{AlP}$ & 1.24 & 1.10 & 1.13 & 1.27 \\
$\mathrm{AlAs}$ & 0.94 & 0.79 & 0.92 & 1.05 \\
$\mathrm{AlSb}$ & 0.35 & 0.26 & 0.53 & 0.45 \\
$\mathrm{GaP}$ & 0.65 & 0.52 & 0.83 & 0.81 \\
$\mathrm{GaAs}$ & 0.35 & 0.35 & 0.52 & 0.50 \\
$\mathrm{GaSb}$ & -0.18 & -0.18 & 0.16 & 0.07 \\
$\mathrm{InP}$ & 0.72 & 0.64 & 0.86 & 0.76 \\
$\mathrm{InAs}$ & 0.40 & 0.37 & 0.50 & 0.50 \\
$\mathrm{InSb}$ & -0.03 & -0.07 & 0.22 & 0.01 \\
$\mathrm{ZnS}$ & 2.13 & 1.98 & 2.05 & 2.22 \\
$\mathrm{ZnSe}$ & 1.66 & 1.55 & 1.48 & 1.70 \\
$\mathrm{ZnTe}$ & 0.89 & 0.81 & 1.00 & 0.84 \\
$\mathrm{CdS}$ & 1.94 & 1.86 & 1.93 & \\
$\mathrm{CdSe}$ & 1.55 & 1.46 & 1.53 & \\
$\mathrm{CdTe}$ & 0.98 & 0.86 & 1.12 & 0.85 \\
$\mathrm{ZnO}$ & 3.54 & 3.31 & & \\
$\mathrm{GaN}$ & 2.45 & 2.28 & 2.37 & \\
\hline \hline
\end{tabular}

LDA, where band gaps were also adjusted to match the experimental values via a rigid shift of the conduction bands [94-96]. The present branch point energies are generally close to these reported values and $G_{0} W_{0} @$ HSE03 values reported for $\mathrm{GaN}$ and $\mathrm{ZnO}$ [15]. We find that branch point energies obtained using Eq. (3) become consistently higher by $0.1-0.4 \mathrm{eV}$ in the 21 semiconductors $(0.1-0.2 \mathrm{eV}$ for 17 semiconductors other than $\mathrm{C}, \mathrm{BN}, \mathrm{GaN}$, and $\mathrm{ZnO}$ ) when only the highest valence band is sampled $\left(N_{\mathrm{VB}}=1\right)$ instead of $N_{\mathrm{VB}}=2$ in combination with the lowest conduction band $\left(N_{\mathrm{CB}}=1\right)$ (number of bands doubled for wurtzite $\mathrm{GaN}$ and $\mathrm{ZnO}$ ). This affects the band alignment by typically $\sim 0.1$ and $\sim 0.3 \mathrm{eV}$ at most.

The IP differences are closer to the actual offset values on the diagonal line when the IP for the (111) $2 \times 1$ surface is used for $\mathrm{Si}$ and Ge instead of the (110) surface. The IP difference between the BN (110) and C (111) $2 \times 1$ surfaces $(1.82 \mathrm{eV})$ also agrees with the interfacial band offset for the $\mathrm{BN} / \mathrm{C}(110)$ interface $(1.86 \mathrm{eV})$ within $0.04 \mathrm{eV}$, while the use of the (110) surfaces for both $\mathrm{BN}$ and $\mathrm{C}$ gives a value of $1.13 \mathrm{eV}$, deviating by $0.73 \mathrm{eV}$ from the interfacial offset. It appears that the more stable (111) surfaces serve as better references for the prediction of interfacial band offsets involving $\mathrm{C}, \mathrm{Si}$, and Ge. Surface-plane dependencies are much smaller for the IPs of $\mathrm{GaN}$ and $\mathrm{ZnO}$ as shown in Fig. 2. The deviations of IP differences from interfacial valence band offsets in $\mathrm{GaN} / \mathrm{ZnO}$ are -0.26 and $-0.36 \mathrm{eV}$ for $(10 \overline{1} 0)$ and $(1 \overline{1} 20)$ surfaces, respectively. The overall performance of the IP-based alignment is also reasonably good. The mean absolute error is $0.16 \mathrm{eV}$ when using the (111) $2 \times 1$ surfaces for $\mathrm{Si}$ and $\mathrm{Ge}$ (and $0.20 \mathrm{eV}$ using the (110) surfaces for all semiconductors). However, its predictive power is more system dependent than the average interfacial offset-based alignment. The IP-based alignment has a tendency to show large errors for interfaces involving antimonides, in particular those with $\mathrm{Ge}$ and $\mathrm{Si}$ as recognized in Figs. 6(a) and 6(b), and the maximum deviation is as large as $0.67 \mathrm{eV}$ [0.85 eV using the (110) surfaces for all semiconductors].

In summary, the branch point energy- and IP-based alignments, which require only bulk and surface plus bulk calculations, respectively, can predict interfacial band offsets reasonably well with mean absolute errors of $\sim 0.1$ and $\sim 0.2 \mathrm{eV}$, respectively. The average interfacial offset-based alignment performs better, but it requires a large set of interface calculations to obtain an alignment with a good transitivity. Even in this case, the maximum deviation is as large as $0.3 \mathrm{eV}$, and therefore explicit calculations of the interfaces of interest are desired, if accurate band offset values are needed.

\section{CONCLUSIONS}

The ionization potentials (IPs) and electron affinities (EAs) as well as the valence and conduction band offsets are determined for 21 semiconductors from group IV, III-V, and II-VI via calculations of actual surfaces and a vast number of heterointerfaces, respectively. The applied methods cover two approximations to DFT, namely the PBE semilocal functional and the HSE hybrid functional, as well as three approximations to MBPT including $G W_{0} @ \mathrm{PBE}, G W^{\mathrm{TC}-\mathrm{TC}} @ \mathrm{HSE}$, and $G W \Gamma^{1} @$ HSE. The experimental IPs, EAs, and band offsets are best reproduced by $G W \Gamma^{1}$ for most semiconductor surfaces and interfaces considered in this study. Subsequently we have evaluated how well more approximate DFT and $G W$ flavors perform compared to $G W \Gamma^{1}$. We find that, although errors in the IP and EA can be large using PBE and HSE, in particular for group II-VI semiconductors, significant error cancellation occurs in the evaluation of the relative band positions or band offsets. As a ground state theory, one expects DFT to predict reasonable valence bands but unreliable conduction bands. Contrary to this common belief, PBE shows smaller deviations in the EA than the IP from experiment and our best theoretical estimates, $G W \Gamma^{1}$. Smaller deviations are also found in the relative CBM positions or conduction band offsets than in the relative VBM positions or valence band offsets. These tendencies are particularly evident for group II-VI semiconductors with wide band gaps. The more accurate $G W_{0} @ \mathrm{PBE}$ and $G W^{\mathrm{TC}-\mathrm{TC}}$ values show small, almost constant deviations in the IP and EA from $G W \Gamma^{1}$ for most semiconductors. They are, therefore, almost on par with $G W \Gamma^{1}$ in predicting the relative band positions.

Since actual interface calculations might be too expensive, we have also evaluated how well the band offsets at the interfaces can be predicted using commonly adopted approximate methods. To this end, interfacial valence band offsets are predicted using band alignments based on the average 
interfacial band offsets, branch point energies, and the IP differences all evaluated at the $G W \Gamma^{1}$ level. The average offset-based alignment shows a small mean absolute error of $0.1 \mathrm{eV}$ and a maximum deviation of $0.3 \mathrm{eV}$, despite the fact that the band offsets are, in principle, interfacial dipole dependent. In other words, a good transitivity is observed for the considered heterointerfaces. The use of the branch point energies also predicts band offsets rather well for most of the interfaces, with a mean absolute error of $0.1 \mathrm{eV}$ and a maximum deviation of $0.4 \mathrm{eV}$. The alignment using the IP differences predicts band offsets almost on par with the branch point energy-based alignment for most of the interfaces. The IP-based alignment, however, depends on the choice of the surface orientation for $\mathrm{C}, \mathrm{Si}$, and $\mathrm{Ge}$. Furthermore, the maximum deviation is as large as $0.7 \mathrm{eV}$, even when the (111) $2 \times 1$ surfaces that reduce the deviations are used. Considering the sizable maximum deviations in the three transitive band alignments, materials screening using simple band alignment procedures should be made with some caution. If accurate interfacial band offsets are needed, an actual calculation of the interface is indispensable.

\section{ACKNOWLEDGMENTS}

This work was supported by the MEXT Elements Strategy Initiative to Form Core Research Center, Grants-in-Aid for Challenging Exploratory Research (Grant No. 26630316) and Scientific Research on Innovative Areas (Grant No. 25106005) from JSPS, the Austrian Science Fund (FWF) within the SFB ViCoM (Grant No. F41) and SFB IR-ON (Grant No. F25), and the Austrian Academy of Sciences within APART. Supercomputing time on the ACCMS at Kyoto University and the Vienna Scientific cluster (VSC) is gratefully acknowledged.
[1] E. T. Yu, J. O. McCaldin, and T. C. McGill, in Solid State Physics, edited by E. Henry and T. David (Academic, Boston, 1992), p. 1.

[2] A. Franciosi and C. G. Van de Walle, Surf. Sci. Rep. 25, 1 (1996).

[3] W. Mönch, Electronic Properties of Semiconductor Interfaces (Springer, Berlin, 2010).

[4] J. Robertson, J. Vac. Sci. Technol. A 31, 050821 (2013).

[5] H. Hosono, in Transparent Electronics From Synthesis to Applications, edited by A. Facchetti and T. J. Marks (John Wiley and Sons, Chichester, UK, 2010), p. 31.

[6] W. A. Harrison, J. Vac. Sci. Technol. 14, 1016 (1977).

[7] C. G. Van de Walle and R. M. Martin, Phys. Rev. B 35, 8154 (1987).

[8] J. Tersoff, Phys. Rev. B 30, 4874 (1984).

[9] J. Tersoff, Phys. Rev. B 32, 6968 (1985).

[10] F. Flores and C. Tejedor, J. Phys. C 12, 731 (1979).

[11] M. Cardona and N. E. Christensen, Phys. Rev. B 35, 6182 (1987).

[12] W. R. L. Lambrecht, B. Segall, and O. K. Andersen, Phys. Rev. B 41, 2813 (1990).

[13] Y.-H. Li, A. Walsh, S. Chen, W.-J. Yin, J.-H. Yang, J. Li, J. L. F. Da Silva, X. G. Gong, and S.-H. Wei, Appl. Phys. Lett. 94, 212109 (2009).

[14] W. Mönch, J. Appl. Phys. 80, 5076 (1996).

[15] A. Schleife, F. Fuchs, C. Rödl, J. Furthmüller, and F. Bechstedt, Appl. Phys. Lett. 94, 012104 (2009).

[16] C. G. Van de Walle and J. Neugebauer, Nature (London) 423, 626 (2003)

[17] L. A. Ledebo and B. K. Ridley, J. Phys. C 15, L961 (1982).

[18] M. J. Caldas, A. Fazzio, and A. Zunger, Appl. Phys. Lett. 45, 671 (1984).

[19] Y. Li, L. E. O’Leary, N. S. Lewis, and G. Galli, J. Phys. Chem. C 117, 5188 (2013).

[20] V. Stevanovic, S. Lany, D. S. Ginley, W. Tumas, and A. Zunger, PCCP 16, 3706 (2014).

[21] A. Grüneis, G. Kresse, Y. Hinuma, and F. Oba, Phys. Rev. Lett. 112, 096401 (2014).
[22] B. Höffling, A. Schleife, C. Rödl, and F. Bechstedt, Phys. Rev. B 85, 035305 (2012).

[23] B.-C. Huang, Y.-P. Chiu, P.-C. Huang, W.-C. Wang, V. T. Tra, J.-C. Yang, Q. He, J.-Y. Lin, C.-S. Chang, and Y.-H. Chu, Phys. Rev. Lett. 109, 246807 (2012).

[24] Y. Hinuma, F. Oba, and I. Tanaka, Phys. Rev. B 88, 075319 (2013).

[25] M. Peressi, N. Binggeli, and A. Baldereschi, J. Phys. D: Appl. Phys. 31, 1273 (1998).

[26] K. Steiner, W. Chen, and A. Pasquarello, Phys. Rev. B 89, 205309 (2014).

[27] J. W. Matthews and A. E. Blakeslee, J. Cryst. Growth 27, 118 (1974).

[28] R. L. Anderson, Solid-State Electron. 5, 341 (1962).

[29] Y. Hinuma, F. Oba, Y. Nose, and I. Tanaka, J. Appl. Phys. 114, 043718 (2013).

[30] J. Robertson and S. J. Clark, Phys. Rev. B 83, 075205 (2011).

[31] A. Walsh and C. R. A. Catlow, J. Mater. Chem. 20, 10438 (2010).

[32] Y. Hinuma, F. Oba, Y. Kumagai, and I. Tanaka, Phys. Rev. B 86, 245433 (2012)

[33] S. H. Vosko, L. Wilk, and M. Nusair, Can. J. Phys. 58, 1200 (1980).

[34] J. P. Perdew and A. Zunger, Phys. Rev. B 23, 5048 (1981).

[35] J. P. Perdew, K. Burke, and M. Ernzerhof, Phys. Rev. Lett. 77, 3865 (1996)

[36] J. P. Perdew, K. Burke, and M. Ernzerhof, Phys. Rev. Lett. 78, 1396 (1997).

[37] A. D. Becke, Phys. Rev. A 38, 3098 (1988).

[38] P. Hohenberg and W. Kohn, Phys. Rev. 136, B864 (1964).

[39] A. V. Krukau, O. A. Vydrov, A. F. Izmaylov, and G. E. Scuseria, J. Chem. Phys. 125, 224106 (2006).

[40] J. Paier, M. Marsman, K. Hummer, G. Kresse, I. C. Gerber, and J. G. Angyan, J. Chem. Phys. 124, 154709 (2006).

[41] F. Oba, M. Choi, A. Togo, and I. Tanaka, Sci. Technol. Adv. Mater. 12, 034302 (2011) 
[42] E. R. Batista, J. Heyd, R. G. Hennig, B. P. Uberuaga, R. L. Martin, G. E. Scuseria, C. J. Umrigar, and J. W. Wilkins, Phys. Rev. B 74, 121102 (2006).

[43] F. Oba, A. Togo, I. Tanaka, J. Paier, and G. Kresse, Phys. Rev. B 77, 245202 (2008).

[44] A. Alkauskas, P. Broqvist, and A. Pasquarello, Phys. Rev. Lett. 101, 046405 (2008).

[45] P. Ágoston, K. Albe, R. M. Nieminen, and M. J. Puska, Phys. Rev. Lett. 103, 245501 (2009).

[46] F. Oba, A. Togo, I. Tanaka, K. Watanabe, and T. Taniguchi, Phys. Rev. B 81, 075125 (2010).

[47] A. Janotti, J. B. Varley, P. Rinke, N. Umezawa, G. Kresse, and C. G. Van de Walle, Phys. Rev. B 81, 085212 (2010).

[48] D. West, Y. Y. Sun, and S. B. Zhang, Appl. Phys. Lett. 101, 082105 (2012).

[49] M. Choi, F. Oba, Y. Kumagai, and I. Tanaka, Adv. Mater. 25, 86 (2013).

[50] H. Peng, D. O. Scanlon, V. Stevanovic, J. Vidal, G. W. Watson, and S. Lany, Phys. Rev. B 88, 115201 (2013).

[51] P. G. Moses, M. Miao, Q. Yan, and C. G. Van de Walle, J. Chem. Phys. 134, 084703 (2011).

[52] A. Alkauskas, P. Broqvist, F. Devynck, and A. Pasquarello, Phys. Rev. Lett. 101, 106802 (2008).

[53] Y. Hinuma, F. Oba, Y. Kumagai, and I. Tanaka, Phys. Rev. B 88, 035305 (2013).

[54] A. Wadehra, J. W. Nicklas, and J. W. Wilkins, Appl. Phys. Lett. 97, 092119 (2010)

[55] L. Hedin, Phys. Rev. 139, A796 (1965).

[56] M. S. Hybertsen and S. G. Louie, Phys. Rev. B 34, 5390 (1986).

[57] X. Zhu and S. G. Louie, Phys. Rev. B 43, 14142 (1991).

[58] R. Shaltaf, G. M. Rignanese, X. Gonze, F. Giustino, and A. Pasquarello, Phys. Rev. Lett. 100, 186401 (2008).

[59] C. Mitra, B. Lange, C. Freysoldt, and J. Neugebauer, Phys. Rev. B 84, 193304 (2011).

[60] A. Punya and W. R. L. Lambrecht, Phys. Rev. B 88, 075302 (2013).

[61] M. Usuda, N. Hamada, T. Kotani, and M. van Schilfgaarde, Phys. Rev. B 66, 125101 (2002).

[62] M. Shishkin, M. Marsman, and G. Kresse, Phys. Rev. Lett. 99, 246403 (2007).

[63] T. L. Gilbert, Phys. Rev. B 12, 2111 (1975).

[64] A. Seidl, A. Görling, P. Vogl, J. A. Majewski, and M. Levy, Phys. Rev. B 53, 3764 (1996).

[65] P. E. Blöchl, Phys. Rev. B 50, 17953 (1994).

[66] J. Heyd, G. Scuseria, and M. Ernzerhof, J. Chem. Phys. 118, 8207 (2003).

[67] J. Heyd and G. Scuseria, J. Chem. Phys. 120, 7274 (2004).

[68] G. Kresse and J. Hafner, Phys. Rev. B 48, 13115 (1993).

[69] G. Kresse and J. Furthmüller, Phys. Rev. B 54, 11169 (1996).

[70] G. Kresse and D. Joubert, Phys. Rev. B 59, 1758 (1999).

[71] J. Klimeš, M. Kaltak, and G. Kresse, Phys. Rev. B 90, 075125 (2014).

[72] C. Friedrich, M. C. Müller, and S. Blügel, Phys. Rev. B 83, 081101 (2011).

[73] J. Paier, M. Marsman, and G. Kresse, Phys. Rev. B 78, 121201 (2008).

[74] S. Lany, J. Osorio-Guillén, and A. Zunger, Phys. Rev. B 75, 241203 (2007)
[75] A. Janotti and C. G. Van de Walle, Phys. Rev. B 75, 121201 (2007).

[76] E. S. Kadantsev and P. Hawrylak, Appl. Phys. Lett. 98, 023108 (2011).

[77] W. Mönch, Semiconductor Surfaces and Interfaces (Springer, Berlin, 2001).

[78] K. Jacobi, G. Zwicker, and A. Gutmann, Surf. Sci. 141, 109 (1984).

[79] P. Lautenschlager, P. B. Allen, and M. Cardona, Phys. Rev. B 31, 2163 (1985).

[80] S. Tsoi, X. Lu, A. K. Ramdas, H. Alawadhi, M. Grimsditch, M. Cardona, and R. Lauck, Phys. Rev. B 74, 165203 (2006).

[81] S. Poncé, G. Antonius, P. Boulanger, E. Cannuccia, A. Marini, M. Côté, and X. Gonze, Comp. Mater. Sci. 83, 341 (2014).

[82] F. Bechstedt, K. Seino, P. H. Hahn, and W. G. Schmidt, Phys. Rev. B 72, 245114 (2005).

[83] S. Botti and M. A. L. Marques, Phys. Rev. Lett. 110, 226404 (2013).

[84] B.-C. Shih, Y. Xue, P. Zhang, M. L. Cohen, and S. G. Louie, Phys. Rev. Lett. 105, 146401 (2010).

[85] W. Chen and A. Pasquarello, Phys. Rev. B 86, 035134 (2012).

[86] W. Chen and A. Pasquarello, Phys. Rev. B 88, 119906(E) (2013).

[87] J. W. Liu, A. Kobayashi, S. Toyoda, H. Kamada, A. Kikuchi, J. Ohta, H. Fujioka, H. Kumigashira, and M. Oshima, Phys. Status Solidi B 248, 956 (2011).

[88] S.-K. Hong, T. Hanada, H. Makino, Y. Chen, H.-J. Ko, T. Yao, A. Tanaka, H. Sasaki, and S. Sato, Appl. Phys. Lett. 78, 3349 (2001).

[89] T. D. Veal et al., Appl. Phys. Lett. 93, 202108 (2008).

[90] E. T. Yu, E. T. Croke, T. C. McGill, and R. H. Miles, Appl. Phys. Lett. 56, 569 (1990).

[91] J. G. Belk, J. L. Sudijono, X. M. Zhang, J. H. Neave, T. S. Jones, and B. A. Joyce, Phys. Rev. Lett. 78, 475 (1997).

[92] R. Jaszek, J. J. Harris, and C. Roberts, J. Appl. Phys. 85, 959 (1999).

[93] F. Oba, F. Ernst, Y. Yu, R. Liu, H. M. Kothari, and J. A. Switzer, J. Am. Ceram. Soc. 88, 253 (2005).

[94] J. Tersoff, Phys. Rev. Lett. 52, 465 (1984).

[95] J. Tersoff, Surf. Sci. 168, 275 (1986).

[96] J. Tersoff, J. Vac. Sci. Technol. B 4, 1066 (1986).

[97] S. Nagao, T. Fujimori, H. Gotoh, H. Fukushima, T. Takano, H. Ito, S. Koshihara, and F. Minami, J. Appl. Phys. 81, 1417 (1997).

[98] J. R. Waldrop, R. W. Grant, and E. A. Kraut, in Proceedings of the 20th Annual Conference on the Physics and Chemistry of Semiconductors Interfaces (AVS, Williamsburg, VA, 1993), p. 1617.

[99] P. Perfetti, F. Patella, F. Sette, C. Quaresima, C. Capasso, A. Savoia, and G. Margaritondo, Phys. Rev. B 30, 4533 (1984).

[100] J. R. Waldrop, S. P. Kowalczyk, R. W. Grant, E. A. Kraut, and D. L. Miller, J. Vac. Sci. Technol. 19, 573 (1981).

[101] J. Batey and S. L. Wright, J. Appl. Phys. 59, 200 (1986).

[102] W. I. Wang and F. Stern, J. Vac. Sci. Technol. B 3, 1280 (1985).

[103] G. J. Gualtieri, G. P. Schwartz, R. G. Nuzzo, and W. A. Sunder, Appl. Phys. Lett. 49, 1037 (1986). 
[104] E. T. Yu, M. C. Phillips, D. H. Chow, D. A. Collins, M. W. Wang, J. O. McCaldin, and T. C. McGill, Phys. Rev. B 46, 13379 (1992).

[105] G. P. Schwartz, G. J. Gualtieri, R. D. Feldman, R. F. Austin, and R. G. Nuzzo, J. Vac. Sci. Technol. B 8, 747 (1990).

[106] M. K. Kelly, D. W. Niles, E. Colavita, G. Margaritondo, and M. Henzler, Appl. Phys. Lett. 46, 768 (1985).

[107] J. R. Waldrop, R. W. Grant, S. P. Kowalczyk, and E. A. Kraut, J. Vac. Sci. Technol. A 3, 835 (1985).

[108] S. P. Kowalczyk, R. W. Grant, J. R. Waldrop, and E. A. Kraut, J. Vac. Sci. Technol. B 1, 684 (1983).

[109] W. Mönch, R. S. Bauer, H. Gant, and R. Murschall, J. Vac. Sci. Technol. 21, 498 (1982).
[110] E. A. Kraut, R. W. Grant, J. R. Waldrop, and S. P. Kowalczyk, Phys. Rev. Lett. 44, 1620 (1980).

[111] J. R. Waldrop, R. W. Grant, and E. A. Kraut, Appl. Phys. Lett. 54, 1878 (1989).

[112] S. P. Kowalczyk, E. A. Kraut, J. R. Waldrop, and R. W. Grant, J. Vac. Sci. Technol. 21, 482 (1982).

[113] E. T. Yu, M. C. Phillips, J. O. McCaldin, and T. C. McGill, J. Vac. Sci. Technol. B 9, 2233 (1991).

[114] W. G. Wilke and K. Horn, J. Vac. Sci. Technol. B 6, 1211 (1988).

[115] K. J. Mackey, P. M. G. Allen, W. G. Herrenden-Harker, R. H. Williams, C. R. Whitehouse, and G. M. Williams, Appl. Phys. Lett. 49, 354 (1986). 